TRANSACTIONS OF THE

AMERICAN MATHEMATICAL SOCIETY

Volume 354, Number 11, Pages 4261-4283

S 0002-9947(02)02987-2

Article electronically published on March 29, 2002

\title{
ASSOCIATED PRIMES OF GRADED COMPONENTS OF LOCAL COHOMOLOGY MODULES
}

\author{
MARKUS P. BRODMANN, MORDECHAI KATZMAN, AND RODNEY Y. SHARP
}

\begin{abstract}
The $i$-th local cohomology module of a finitely generated graded module $M$ over a standard positively graded commutative Noetherian ring $R$, with respect to the irrelevant ideal $R_{+}$, is itself graded; all its graded components are finitely generated modules over $R_{0}$, the component of $R$ of degree 0 . It is known that the $n$-th component $H_{R_{+}}^{i}(M)_{n}$ of this local cohomology module $H_{R_{+}}^{i}(M)$ is zero for all $n>>0$. This paper is concerned with the asymptotic behaviour of $\operatorname{Ass}_{R_{0}}\left(H_{R_{+}}^{i}(M)_{n}\right)$ as $n \rightarrow-\infty$.

The smallest $i$ for which such study is interesting is the finiteness dimension $f$ of $M$ relative to $R_{+}$, defined as the least integer $j$ for which $H_{R_{+}}^{j}(M)$ is not finitely generated. Brodmann and Hellus have shown that $\operatorname{Ass}_{R_{0}}\left(H_{R_{+}}^{f}(M)_{n}\right)$ is constant for all $n<<0$ (that is, in their terminology, $\operatorname{Ass}_{R_{0}}\left(H_{R_{+}}^{f}(M)_{n}\right)$ is asymptotically stable for $n \rightarrow-\infty)$. The first main aim of this paper is to identify the ultimate constant value (under the mild assumption that $R$ is a homomorphic image of a regular ring): our answer is precisely the set of contractions to $R_{0}$ of certain relevant primes of $R$ whose existence is confirmed by Grothendieck's Finiteness Theorem for local cohomology.

Brodmann and Hellus raised various questions about such asymptotic behaviour when $i>f$. They noted that Singh's study of a particular example (in which $f=2)$ shows that $\operatorname{Ass}_{R_{0}}\left(H_{R_{+}}^{3}(R)_{n}\right)$ need not be asymptotically stable for $n \rightarrow-\infty$. The second main aim of this paper is to determine, for Singh's example, $\operatorname{Ass}_{R_{0}}\left(H_{R_{+}}^{3}(R)_{n}\right)$ quite precisely for every integer $n$, and, thereby, answer one of the questions raised by Brodmann and Hellus.
\end{abstract}

\section{INTRODUCTION}

Let $R=\bigoplus_{n \in \mathbb{N}_{0}} R_{n}$ be a positively graded commutative Noetherian ring which is standard in the sense that $R=R_{0}\left[R_{1}\right]$, and set $R_{+}:=\bigoplus_{n \in \mathbb{N}} R_{n}$, the irrelevant ideal of $R$. (Here, $\mathbb{N}_{0}$ and $\mathbb{N}$ denote the set of non-negative and positive integers respectively; $\mathbb{Z}$ will denote the set of all integers.) Let $M=\bigoplus_{n \in \mathbb{Z}} M_{n}$ be a non-zero finitely generated graded $R$-module. This paper is concerned with the behaviour of the graded components of the graded local cohomology modules $H_{R_{+}}^{i}(M)\left(i \in \mathbb{N}_{0}\right)$ of $M$ with respect to $R_{+}$.

Received by the editors November 2, 2001.

2000 Mathematics Subject Classification. Primary 13D45, 13E05, 13A02, 13P10; Secondary $13 \mathrm{C} 15$.

Key words and phrases. Graded commutative Noetherian ring, graded local cohomology module, associated prime ideal, ideal transform, regular ring, Gröbner bases.

The third author was partially supported by the Swiss National Foundation (project number 20-52762.97). 
It is known (see $[\mathrm{B}-\mathrm{S}, 15.1 .5])$ that there exists $r \in \mathbb{Z}$ such that $H_{R_{+}}^{i}(M)_{n}=0$ for all $i \in \mathbb{N}_{0}$ and all $n \geq r$, and that $H_{R_{+}}^{i}(M)_{n}$ is a finitely generated $R_{0}$-module for all $i \in \mathbb{N}_{0}$ and all $n \in \mathbb{Z}$. Set

$$
f:=f_{R_{+}}(M)=\inf \left\{i \in \mathbb{N}: H_{R_{+}}^{i}(M) \text { is not finitely generated }\right\},
$$

the finiteness dimension of $M$ relative to $R_{+}$; see [B-S, 9.1.3]. We assume that $f$ is finite. M. Brodmann and M. Hellus have shown in [B-H, Proposition 5.6] that $\operatorname{Ass}_{R_{0}}\left(H_{R_{+}}^{f}(M)_{n}\right)$ is constant for all $n<<0$. The first part (\$1) of this paper determines the ultimate constant value under the mild restriction that $R$ is a homomorphic image of a regular (commutative Noetherian) ring; the main result is related to Grothendieck's Finiteness Theorem for local cohomology, which (under the specified restriction) gives an alternative description of $f$. Let $* \operatorname{Spec}(R)$ denote the set of graded prime ideals of $R$, and $\operatorname{Proj}(R)$ the set $\left\{\mathfrak{p} \in{ }^{*} \operatorname{Spec}(R): \mathfrak{p} \nsupseteq R_{+}\right\}$. Write

$$
\lambda_{R_{+}}^{R_{+}}(M):=\inf \left\{\operatorname{depth}_{R_{\mathfrak{p}}} M_{\mathfrak{p}}+\operatorname{ht}\left(R_{+}+\mathfrak{p}\right) / \mathfrak{p}: \mathfrak{p} \in \operatorname{Proj}(R)\right\} .
$$

(We interpret the depth of a zero module as $\infty$.) It is a consequence of Grothendieck's Finiteness Theorem [G, Exposé VIII, Corollaire 2.3] that, when $R$ is a homomorphic image of a regular ring,

$$
f=\lambda_{R_{+}}^{R_{+}}(M)=\inf \left\{\operatorname{depth}_{R_{\mathfrak{p}}} M_{\mathfrak{p}}+\operatorname{ht}\left(R_{+}+\mathfrak{p}\right) / \mathfrak{p}: \mathfrak{p} \in \operatorname{Proj}(R)\right\} .
$$

(See [B-S, 13.1.17].) The main result of $§ 1$ is that, under the assumption that $R$ is a homomorphic image of a regular ring,

$$
\begin{aligned}
\{\mathfrak{p} & \left.\cap R_{0}: \mathfrak{p} \in \operatorname{Proj}(R) \text { and } \operatorname{depth}_{R_{\mathfrak{p}}} M_{\mathfrak{p}}+\mathrm{ht}\left(\mathfrak{p}+R_{+}\right) / \mathfrak{p}=f\right\} \\
= & \operatorname{Ass}_{R_{0}}\left(H_{R_{+}}^{f}(M)_{n}\right) \quad \text { for all } n<<0 .
\end{aligned}
$$

The final 92 is concerned with the asymptotic behaviour of $\operatorname{Ass}_{R_{0}}\left(H_{R_{+}}^{i}(M)_{n}\right)$ as $n \rightarrow-\infty$ when $i>f$. Brodmann and Hellus say that $\operatorname{Ass}_{R_{0}}\left(H_{R_{+}}^{i}(M)_{n}\right)$ is asymptotically stable (respectively asymptotically increasing) for $n \rightarrow-\infty$ if there exists $n_{0} \in \mathbb{Z}$ such that $\operatorname{Ass}_{R_{0}}\left(H_{R_{+}}^{i}(M)_{n}\right)=\operatorname{Ass}_{R_{0}}\left(H_{R_{+}}^{i}(M)_{n_{0}}\right)$ (respectively $\left.\operatorname{Ass}_{R_{0}}\left(H_{R_{+}}^{i}(M)_{n}\right) \subseteq \operatorname{Ass}_{R_{0}}\left(H_{R_{+}}^{i}(M)_{n-1}\right)\right)$ for all $n \leq n_{0}$. They used an example of A. Singh [Si, §4] to show that, when $i>f, \operatorname{Ass}_{R_{0}}\left(H_{R_{+}}^{i}(M)_{n}\right)$ need not be asymptotically stable for $n \rightarrow-\infty$. In $\$ 2$ we use Gröbner basis techniques to show that, for Singh's example,

$$
R=\mathbb{Z}[X, Y, Z, U, V, W] /(X U+Y V+Z W),
$$

where the polynomial ring $\mathbb{Z}[X, Y, Z, U, V, W]$ is graded so that its 0 -th component is $\mathbb{Z}[X, Y, Z]$ and $U, V, W$ have degree 1 , we have

$$
\operatorname{Ass}_{R_{0}}\left(H_{R_{+}}^{3}(R)_{-d}\right)=\{(X, Y, Z)\} \cup\{(p, X, Y, Z): p \in \Pi(d-2)\} \quad \text { for all } d \geq 3,
$$

where

$$
\Pi(d-2):=\left\{p: p \text { is a prime factor of }\left(\begin{array}{c}
d-2 \\
i
\end{array}\right) \text { for some } i \in\{0, \ldots, d-2\}\right\} .
$$

It follows that $\operatorname{Ass}_{R_{0}}\left(H_{R_{+}}^{3}(R)_{n}\right)$ is not asymptotically increasing for $n \rightarrow-\infty$, and this settles a question raised by Brodmann and Hellus. 


\section{Asymptotic BehaViour at the Finiteness Dimension}

1.1. Notation. The notation introduced in the above 0 will be maintained for the whole paper. We shall only assume that $R$ is a homomorphic image of a regular ring when this is explicitly stated. Here we introduce additional notation.

We use, for $j \in \mathbb{Z}$, the notation $L_{j}$ to denote the $j$-th component of a $\mathbb{Z}$-graded module $L$, and $(\bullet)(j)$ to denote the $j$-th shift functor on the category of graded $R$-modules and homogeneous homomorphisms (by "homogeneous" here, we mean "homogeneous of degree zero"). It will be convenient to have available the concepts of the end and beginning $(\operatorname{beg}(L))$ of the graded $R$-module $L=\bigoplus_{n \in \mathbb{Z}} L_{n}$, which are defined by

$$
\operatorname{end}(L):=\sup \left\{n \in \mathbb{Z}: L_{n} \neq 0\right\} \quad \text { and } \quad \operatorname{beg}(L):=\inf \left\{n \in \mathbb{Z}: L_{n} \neq 0\right\} .
$$

(Note that end $(L)$ could be $\infty$, and that the supremum of the empty set of integers is to be taken as $-\infty$; similar comments apply to beg $(L)$.)

For $\mathfrak{p} \in \operatorname{Spec}(R)$, we abbreviate $\operatorname{depth}_{R_{\mathfrak{p}}} M_{\mathfrak{p}}$ by $\operatorname{depth} M_{\mathfrak{p}}$ and the projective dimension proj $\operatorname{dim}_{R_{\mathfrak{p}}} M_{\mathfrak{p}}$ by $\operatorname{proj} \operatorname{dim} M_{\mathfrak{p}}$.

1.2. Lemma. The notation is as in 0 and 1.1. Let $\mathfrak{p} \in \operatorname{Proj}(R) \cap \operatorname{Ass}_{R} M$ be such that $\operatorname{ht}\left(\mathfrak{p}+R_{+}\right) / \mathfrak{p}=1$. Set $\mathfrak{p}_{0}=\mathfrak{p} \cap R_{0}$. Then $\mathfrak{p}_{0} \in \operatorname{Ass}_{R_{0}}\left(H_{R_{+}}^{1}(M)_{n}\right)$ for all $n<\operatorname{beg}(M)$.

Proof. Set $\bar{M}:=M / \Gamma_{R_{+}}(M)$, and note that, by [B-S, 2.1.12 and 2.1.7(iii)],

$$
\operatorname{Ass}_{R}(\bar{M})=\operatorname{Proj}(R) \cap \operatorname{Ass}_{R} M
$$

and there is a homogeneous isomorphism $H_{R_{+}}^{1}(M) \cong H_{R_{+}}^{1}(\bar{M})$. We therefore can, and do, assume that $\Gamma_{R_{+}}(M)=0$ in the remainder of this proof.

We now use homogeneous localization at $\mathfrak{p}+R_{+}$to see that it is enough to prove the claim under the additional hypotheses that $R$ is *local with unique *maximal ideal $\mathfrak{m}$, and that $\mathfrak{m}_{0}:=\mathfrak{m} \cap R_{0}=\mathfrak{p}_{0}$. The assumptions that $R$ is standard and *local with $\mathfrak{m}_{0}=\mathfrak{p}_{0}$, and that $\operatorname{ht}\left(\mathfrak{p}+R_{+}\right) / \mathfrak{p}=1$, ensure that there exists $g_{1} \in R_{1} \backslash \mathfrak{p}$, and that, then, $\sqrt{\mathfrak{p}+g_{1} R}=\mathfrak{p}+R_{+}$.

Now there exists $t \in \mathbb{Z}$ such that $M$ has a graded $R$-submodule $N$ homogeneously isomorphic to $(R / \mathfrak{p})(-t)$. We now consider the ideal transform $D_{R g_{1}}(N)$ of $N$ with respect to $R g_{1}$ : this is naturally graded, and since $g_{1}$ is a non-zerodivisor on $R / \mathfrak{p}$, the description of this ideal transform afforded by [B-S, Theorem 2.2.16] shows that (a) multiplication by $g_{1}$ provides a homogeneous isomorphism $D_{R g_{1}}(N) \stackrel{\cong}{\longrightarrow}$ $D_{R g_{1}}(N)(1)$, and that (b) $\mathfrak{p}_{0} \in \operatorname{Ass}_{R_{0}}\left(\left(D_{R g_{1}}(N)_{n}\right)\right.$ for all $n \in \mathbb{Z}$.

Point (a) leads to the conclusion that multiplication by $g_{1}$ provides a homogeneous isomorphism $H_{R_{+}}^{i}\left(D_{R g_{1}}(N)\right) \stackrel{\cong}{\longrightarrow} H_{R_{+}}^{i}\left(D_{R g_{1}}(N)\right)(1)$ for each $i \in \mathbb{N}_{0}$, and so, in particular, for $i=0$ and 1. Since $g_{1} \in R_{+}$, we conclude that $H_{R_{+}}^{i}\left(D_{R g_{1}}(N)\right)=0$ for $i=0,1$.

By [B-S, 2.2.4], the natural (homogeneous) monomorphism $\eta_{N}: N \longrightarrow D_{R g_{1}}(N)$ has cokernel isomorphic to $H_{R g_{1}}^{1}(N)$. But, since $\sqrt{\mathfrak{p}+g_{1} R}=\mathfrak{p}+R_{+}$and $N$ is annihilated by $\mathfrak{p}$, there is a (homogeneous) isomorphism $H_{R g_{1}}^{1}(N) \cong H_{R_{+}}^{1}(N)$. Thus the cokernel of the monomorphism $\eta_{N}$ is $R_{+}$-torsion.

It now follows from $\mathrm{B}-\mathrm{S}, 2.2 .13$ and 12.4.2(ii)] that there is a homogeneous isomorphism $D_{R g_{1}}(N) \cong D_{R_{+}}(N)$. We can thus conclude that $\mathfrak{p}_{0} \in \operatorname{Ass}_{R_{0}}\left(\left(D_{R_{+}}(N)_{n}\right)\right.$ for all $n \in \mathbb{Z}$. We now note that, since $D_{R_{+}}$is a left exact functor, there is a homogeneous $R$-monomorphism $D_{R_{+}}(N) \longrightarrow D_{R_{+}}(M)$; the result now follows from 
the exact sequence

$$
0 \longrightarrow M \longrightarrow D_{R_{+}}(M) \longrightarrow H_{R_{+}}^{1}(M) \longrightarrow 0
$$

of graded $R$-modules and homogeneous $R$-homomorphisms.

For part of the proof of our main result of this section, we shall be able to reduce to the case where $R_{0}$ is a regular local ring and $R=R_{0}\left[X_{1}, \ldots, X_{r}\right]$ is a polynomial ring over $R_{0}$ in which the independent indeterminates $X_{1}, \ldots, X_{r}$ all have degree 1. This explains why several subsequent lemmas are concerned with this case.

1.3. Lemma. The notation is as in $₫ 0$ and 1.1 In addition, suppose that $\left(R_{0}, \mathfrak{m}_{0}\right)$ is a regular local ring of dimension $d$ and that $R=R_{0}\left[X_{1}, \ldots, X_{r}\right]$, a polynomial ring graded in the usual way. Suppose that $\mathfrak{p} \in \operatorname{Supp}(M) \cap \operatorname{Proj}(R)$ is such that $\mathfrak{p} \cap R_{0}=\mathfrak{m}_{0}$. Then

$$
\operatorname{depth} M_{\mathfrak{p}}+\operatorname{ht}\left(\mathfrak{p}+R_{+}\right) / \mathfrak{p}=d+r-\operatorname{proj} \operatorname{dim} M_{\mathfrak{p}} .
$$

Proof. As $R$ is a catenary domain,

$$
\operatorname{ht}\left(\mathfrak{p}+R_{+}\right) / \mathfrak{p}=\operatorname{ht}\left(\mathfrak{p}+R_{+}\right)-\operatorname{ht} \mathfrak{p}=d+r-\operatorname{ht} \mathfrak{p} .
$$

Moreover, by the Auslander-Buchsbaum-Serre Theorem,

$$
\operatorname{depth} M_{\mathfrak{p}}=\operatorname{dim} R_{\mathfrak{p}}-\operatorname{proj} \operatorname{dim} M_{\mathfrak{p}}=\text { ht } \mathfrak{p}-\operatorname{proj} \operatorname{dim} M_{\mathfrak{p}}
$$

1.4. Lemma. The notation is as in $₫ 0$ and 1.1 . In addition, suppose that $\left(R_{0}, \mathfrak{m}_{0}\right)$ is a regular local ring of dimension $d$ and that $R=R_{0}\left[X_{1}, \ldots, X_{r}\right]$, a polynomial ring graded in the usual way.

Let $\left(R_{0}^{\prime}, \mathfrak{m}_{0}^{\prime}\right)$ be a regular local flat extension ring of $R_{0}$ such that $\mathfrak{m}_{0} R_{0}^{\prime}=\mathfrak{m}_{0}^{\prime}$. Let $R^{\prime}=R \otimes_{R_{0}} R_{0}^{\prime}$, which we identify with $R_{0}^{\prime}\left[X_{1}, \ldots, X_{r}\right]$ in the obvious way. Let $M^{\prime}$ denote the finitely generated graded $R^{\prime}$-module $M \otimes_{R} R^{\prime}$, and let $\mathfrak{p}^{\prime} \in \operatorname{Proj}\left(R^{\prime}\right)$ be such that $\mathfrak{p}^{\prime} \cap R_{0}^{\prime}=\mathfrak{m}_{0}^{\prime}$. Set $\mathfrak{p}:=\mathfrak{p}^{\prime} \cap R$. Then $\mathfrak{p} \in \operatorname{Proj}(R)$ and $\mathfrak{p} \cap R_{0}=\mathfrak{m}_{0}$; also

$$
\operatorname{depth} M_{\mathfrak{p}}+\operatorname{ht}\left(\mathfrak{p}+R_{+}\right) / \mathfrak{p} \leq \operatorname{depth}_{R_{\mathfrak{p}^{\prime}}^{\prime}} M_{\mathfrak{p}^{\prime}}^{\prime}+\mathrm{ht}\left(\mathfrak{p}^{\prime}+R_{+}^{\prime}\right) / \mathfrak{p}^{\prime}
$$

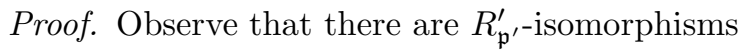

$$
M_{\mathfrak{p}^{\prime}}^{\prime} \cong\left(M \otimes_{R} R^{\prime}\right) \otimes_{R^{\prime}} R_{\mathfrak{p}^{\prime}}^{\prime} \cong M \otimes_{R} R_{\mathfrak{p}^{\prime}}^{\prime} \cong M_{\mathfrak{p}} \otimes_{R_{\mathfrak{p}}} R_{\mathfrak{p}^{\prime}}^{\prime}
$$

As $R_{\mathfrak{p}^{\prime}}^{\prime}$ is a flat $R_{\mathfrak{p}}$-algebra, $\operatorname{proj} \operatorname{dim} M_{\mathfrak{p}^{\prime}}^{\prime} \leq \operatorname{proj} \operatorname{dim} M_{\mathfrak{p}}$. Hence, by two uses of Lemma 1.3.

$$
\begin{aligned}
\operatorname{depth} M_{\mathfrak{p}}+\operatorname{ht}\left(\mathfrak{p}+R_{+}\right) / \mathfrak{p} & =d+r-\operatorname{proj} \operatorname{dim} M_{\mathfrak{p}} \\
& \leq d+r-\operatorname{proj} \operatorname{dim} M_{\mathfrak{p}^{\prime}}^{\prime} \\
& =\operatorname{depth} M_{\mathfrak{p}^{\prime}}^{\prime}+\operatorname{ht}\left(\mathfrak{p}^{\prime}+R_{+}^{\prime}\right) / \mathfrak{p}^{\prime}
\end{aligned}
$$

1.5. Lemma. The notation is as in $\oint_{0}$ and 1.1 In addition, suppose that $\left(R_{0}, \mathfrak{m}_{0}\right)$ is a regular local ring of dimension d such that the field $R_{0} / \mathfrak{m}_{0}$ is algebraically closed and that $R=R_{0}\left[X_{1}, \ldots, X_{r}\right]$, a polynomial ring graded in the usual way. 
Suppose that $r>1$, that $f_{R_{+}}(M)=r$ and that $\mathfrak{m}_{0} \in \operatorname{Ass}_{R_{0}}\left(H_{R_{+}}^{r}(M)_{n}\right)$ for all $n<<0$. Then there exists $y \in R_{1} \backslash \mathfrak{m}_{0} R_{1}$ such that $y$ is a non-zerodivisor on $M / \Gamma_{R_{+}}(M)$, that $f_{R_{+}}(M / y M)=r-1$ and that

$$
\mathfrak{m}_{0} \in \operatorname{Ass}_{R_{0}}\left(H_{R_{+}}^{r-1}(M / y M)_{n}\right) \quad \text { for all } n<<0 .
$$

Proof. Set $\bar{M}:=M / \Gamma_{R_{+}}(M)$. For a homogeneous element $y$ of $R$, we have homogeneous isomorphisms

$$
\bar{M} / y \bar{M} \cong M /\left(y M+\Gamma_{R_{+}}(M)\right) \cong(M / y M) /\left(\left(y M+\Gamma_{R_{+}}(M)\right) / y M\right),
$$

so that there are homogeneous isomorphisms

$$
H_{R_{+}}^{i}(M) \cong H_{R_{+}}^{i}(\bar{M}) \text { and } H_{R_{+}}^{i}(M / y M) \cong H_{R_{+}}^{i}(\bar{M} / y \bar{M})
$$

for all $i>0$. We may therefore replace $M$ by $\bar{M}$. We therefore assume that $\Gamma_{R_{+}}(M)=0$ and $\operatorname{Ass}_{R} M \subseteq \operatorname{Proj}(R)$.

Now let $\mathfrak{p} \in \operatorname{Ass}_{R} M$ and set $\mathfrak{p}_{0}:=\mathfrak{p} \cap R_{0}$. Then, since $R$ is a regular, and therefore catenary, domain,

$$
\begin{aligned}
\operatorname{ht}_{R_{0}} \mathfrak{p}_{0}+r-\operatorname{ht} \mathfrak{p} & =\operatorname{ht}\left(\mathfrak{p}_{0} R+R_{+}\right)-\operatorname{ht} \mathfrak{p} \\
& =\operatorname{ht}\left(\mathfrak{p}_{0} R+R_{+}\right) / \mathfrak{p}=\operatorname{ht}\left(\mathfrak{p}+R_{+}\right) / \mathfrak{p} \\
& =\operatorname{depth} M_{\mathfrak{p}}+\operatorname{ht}\left(\mathfrak{p}+R_{+}\right) / \mathfrak{p} \\
& \geq f_{R_{+}}(M)=r .
\end{aligned}
$$

(We have used Grothendieck's Finiteness Theorem to obtain the inequality.) Therefore ht $\mathfrak{p}_{0} R=\mathrm{ht}_{R_{0}} \mathfrak{p}_{0}=\mathrm{ht} \mathfrak{p}$, so that $\mathfrak{p}=\mathfrak{p}_{0} R$ and $\mathfrak{p} \subseteq \mathfrak{m}_{0} R$. It therefore follows that, if we let $U$ denote the subset of $R_{1} \backslash \mathfrak{m}_{0} R_{1}$ defined by

$$
U:=\left\{a_{1} X_{1}+a_{2} X_{2}:\left(a_{1}, a_{2}\right) \in R_{0} \times R_{0} \backslash\left(\mathfrak{m}_{0} \times \mathfrak{m}_{0}\right)\right\},
$$

then $U \cap \mathfrak{p}=\emptyset$. Therefore each element of $U$ is a non-zerodivisor on $M$.

Set $J:=\Gamma_{\mathfrak{m}_{0} R}\left(H_{R_{+}}^{r}(M)\right)=\bigoplus_{n \in \mathbb{Z}} \Gamma_{\mathfrak{m}_{0}}\left(H_{R_{+}}^{r}(M)_{n}\right)$. The hypotheses ensure that $J$ is not a finitely generated $R$-module. We shall show that one of the elements of $U$ can be taken for $y$. To achieve this, we suppose that, for all $x \in U$, there exists $n_{x} \in \mathbb{Z}$ such that, for all $n \leq n_{x}$, it is the case that $\mathfrak{m}_{0} \notin \operatorname{Ass}_{R_{0}}\left(H_{R_{+}}^{r-1}(M / x M)_{n}\right)$, and we seek a contradiction.

This supposition means that, for each $x \in U$, we have $\Gamma_{\mathfrak{m}_{0}}\left(H_{R_{+}}^{r-1}(M / x M)_{n}\right)=0$ for all $n \leq n_{x}$. Since $f_{R_{+}}(M)=r$, there exists $\widetilde{n} \in \mathbb{Z}$ such that $H_{R_{+}}^{r-1}(M)_{n}=0$ for all $n \leq \widetilde{n}$. For each $x \in U$, the application of local cohomology with respect to $R_{+}$ to the exact sequence

$$
0 \longrightarrow M(-1) \stackrel{x}{\longrightarrow} M \longrightarrow M / x M \longrightarrow 0
$$

shows that $f_{R_{+}}(M / x M) \geq r-1$ and leads to an exact sequence of $R_{0}$-modules

$$
0 \longrightarrow H_{R_{+}}^{r-1}(M / x M)_{n} \longrightarrow H_{R_{+}}^{r}(M)_{n-1} \stackrel{x}{\longrightarrow} H_{R_{+}}^{r}(M)_{n}
$$

for each $n \leq \widetilde{n}$. The left exactness of the functor $\Gamma_{\mathfrak{m}_{0}}$ therefore leads to the conclusion that, for each $x \in U$, the map

$$
J_{n-1}=\Gamma_{\mathfrak{m}_{0}}\left(H_{R_{+}}^{r}(M)_{n-1}\right) \stackrel{x}{\longrightarrow} J_{n}=\Gamma_{\mathfrak{m}_{0}}\left(H_{R_{+}}^{r}(M)_{n}\right)
$$

is injective for all $n \leq \min \left\{\tilde{n}, n_{x}\right\}$. Hence $\left(0:_{J} x\right)$ is an $R$-module of finite length, for all $x \in U$. Since $R_{0} / \mathfrak{m}_{0}$ is algebraically closed, we can now deduce from $\mathrm{B}$ Corollary (2.2)] that $J$ is an $R$-module of finite length, and this is a 
contradiction. We have therefore proved that there exists $y \in U$ such that $\mathfrak{m}_{0} \in$ $\operatorname{Ass}_{R_{0}}\left(H_{R_{+}}^{r-1}(M / y M)_{n}\right)$ for infinitely many $n<0$. This implies that $f_{R_{+}}(M / y M) \leq$ $r-1$; therefore, as we have already noted that $f_{R_{+}}(M / y M) \geq r-1$, we must have $f_{R_{+}}(M / y M)=r-1$. Hence, by [B-H], Proposition (5.6)], $\operatorname{Ass}_{R_{0}}\left(H_{R_{+}}^{r-1}(M / y M)_{n}\right)$ is asymptotically stable for $n \rightarrow-\infty$; therefore $\mathfrak{m}_{0} \in \operatorname{Ass}_{R_{0}}\left(H_{R_{+}}^{r-1}(M / y M)_{n}\right)$ for all $n<<0$.

1.6. Lemma. The notation is as in 90 and 1.1 In addition, suppose that $\left(R_{0}, \mathfrak{m}_{0}\right)$ is a regular local ring of dimension $d$ and that $R=R_{0}\left[X_{1}, \ldots, X_{r}\right]$, a polynomial ring graded in the usual way.

Assume that $f_{R_{+}}(M)<r$ and that $0 \rightarrow N \rightarrow F \rightarrow M \rightarrow 0$ is an exact sequence of finitely generated graded $R$-modules and homogeneous homomorphisms in which $F$ is free. Then:

(i) depth $N_{\mathfrak{p}}=\min \left\{\right.$ ht $\mathfrak{p}$, depth $\left.M_{\mathfrak{p}}+1\right\}$ for all $\mathfrak{p} \in \operatorname{Supp}(N)$;

(ii) for $i \in \mathbb{N}_{0}$, the (necessarily homogeneous) connecting homomorphism

$$
H_{R_{+}}^{i}(M) \rightarrow H_{R_{+}}^{i+1}(N)
$$

induced by the given exact sequence is an isomorphism when $i<r-1$ and a monomorphism when $i=r-1$; and

(iii) $f_{R_{+}}(N)=f_{R_{+}}(M)+1$.

Proof. Note that $N \neq 0$ because $f_{R_{+}}(F)=r$.

(i) This is immediate from the exact sequence $0 \rightarrow N_{\mathfrak{p}} \rightarrow F_{\mathfrak{p}} \rightarrow M_{\mathfrak{p}} \rightarrow 0$.

(ii) This is immediate from the fact that $H_{R_{+}}^{i}(F)=0$ for all $i<r$.

(iii) This now follows from part (ii) and the hypothesis that $f_{R_{+}}(M)<r$.

1.7. Lemma. Assume that $\left(R_{0}, \mathfrak{m}_{0}\right)$ is a regular local ring. Then there exists a regular local flat extension ring $\left(R_{0}^{\prime}, \mathfrak{m}_{0}^{\prime}\right)$ of $R_{0}$ such that $\mathfrak{m}_{0} R_{0}^{\prime}=\mathfrak{m}_{0}^{\prime}$ and $R_{0}^{\prime} / \mathfrak{m}_{0}^{\prime}$ is algebraically closed.

Proof. Denote as usual $\operatorname{dim} R_{0}$ by $d$. Let $\left(\widehat{R_{0}}, \widehat{\mathfrak{m}_{0}}\right)$ denote the completion of $R_{0}$, so that $\widehat{\mathfrak{m}_{0}}=\mathfrak{m}_{0} \widehat{R_{0}}$; of course, this is a regular local flat extension ring of $R_{0}$ of dimension $d$. By [B-M-M, Proposition (2.2)], there exists a (Noetherian) local flat extension ring $\left(R_{0}^{\prime}, \mathfrak{m}_{0}^{\prime}\right)$ of $\widehat{R_{0}}$ such that $\widehat{\mathfrak{m}_{0}} R_{0}^{\prime}=\mathfrak{m}_{0}^{\prime}$ and $R_{0}^{\prime} / \mathfrak{m}_{0}^{\prime}$ is algebraically closed. Therefore $\mathfrak{m}_{0} R_{0}^{\prime}=\mathfrak{m}_{0}^{\prime}$, so that $\mathfrak{m}_{0}^{\prime}$ can be generated by $d$ elements. By flatness, $\operatorname{dim} R_{0}^{\prime} \geq d$, and so $\left(R_{0}^{\prime}, \mathfrak{m}_{0}^{\prime}\right)$ is a regular local ring of dimension $d$.

We are now ready to present our main result of this section.

1.8. Theorem. Assume that the graded ring $R$ is a homomorphic image of a regular (commutative Noetherian) ring, and that the non-zero graded $R$-module $M=\bigoplus_{n \in \mathbb{Z}} M_{n}$ is finitely generated and not $R_{+}$-torsion. Set

$$
f:=f_{R_{+}}(M)=\inf \left\{i \in \mathbb{N}: H_{R_{+}}^{i}(M) \text { is not finitely generated }\right\} .
$$

Then

$$
\operatorname{Ass}_{R_{0}}\left(H_{R_{+}}^{f}(M)_{n}\right)=\left\{\mathfrak{p} \cap R_{0}: \mathfrak{p} \in \operatorname{Proj}(R) \text { and } \operatorname{depth} M_{\mathfrak{p}}+\mathrm{ht}\left(\mathfrak{p}+R_{+}\right) / \mathfrak{p}=f\right\}
$$

for all $n<<0$.

Note. By Grothendieck's Finiteness Theorem (see [B-S, 13.1.17]), the set on the right-hand side of the final display in the statement of the theorem is non-empty: note that $f$ is finite. A consequence of this theorem is that that set is finite. 
Proof. We first show by induction on $f$ that, for $\mathfrak{p} \in \operatorname{Proj}(R)$ with

$$
\operatorname{depth} M_{\mathfrak{p}}+\operatorname{ht}\left(\mathfrak{p}+R_{+}\right) / \mathfrak{p}=f
$$

we have $\mathfrak{p} \cap R_{0} \in \operatorname{Ass}_{R_{0}}\left(H_{R_{+}}^{f}(M)_{n}\right)$ for all $n<<0$. Now $\operatorname{ht}\left(\mathfrak{p}+R_{+}\right) / \mathfrak{p} \geq 1$; so, if $f=1$ and

$$
\operatorname{depth} M_{\mathfrak{p}}+\operatorname{ht}\left(\mathfrak{p}+R_{+}\right) / \mathfrak{p}=1,
$$

then $\mathfrak{p} \in \operatorname{Ass}_{R} M$. The claim in the case when $f=1$ is therefore immediate from Lemma 1.2

Thus we assume now that $f>1$ and make the obvious inductive assumption. One can use homogeneous localization at $\mathfrak{p}+R_{+}$to see that it is enough to complete the inductive step under the additional hypotheses that $R$ is *local with unique *maximal ideal $\mathfrak{m}$, and that $\mathfrak{m}_{0}:=\mathfrak{m} \cap R_{0}=\mathfrak{p}_{0}$.

Set $\bar{M}:=M / \Gamma_{R_{+}}(M)$; recall $\left.(\underline{\mathrm{B}-\mathrm{S}}, 2.1 .7]\right)$ that there are homogeneous isomorphisms $H_{R_{+}}^{i}(M) \stackrel{\cong}{\longrightarrow} H_{R_{+}}^{i}(\bar{M})$ for each $i \in \mathbb{N}$. Since $M_{\mathfrak{p}} \cong \bar{M}_{\mathfrak{p}}$, it follows that one may assume, in this inductive step, that $\Gamma_{R_{+}}(M)=0$.

The argument now splits into two cases, according as $\mathfrak{p} \in \operatorname{Ass}_{R} M$ or $\mathfrak{p} \notin \operatorname{Ass}_{R} M$. In the first case, it follows from [B-S, 15.1.2] that there exist a positive integer $d$ and a homogeneous element $g_{d} \in R_{d}$ which is a non-zerodivisor on $M$. Let $\mathfrak{q}$ be a minimal prime ideal of $\mathfrak{p}+R g_{d}$; necessarily, $\mathfrak{q} \in \operatorname{Proj}(R)$ (since $f>1$ ), and $\mathfrak{q} \cap R_{0}=\mathfrak{m}_{0}$. The catenarity of $R$ ensures that ht $\left(\left(\mathfrak{q}+R_{+}\right) / \mathfrak{q}\right)=f-1$. It follows from [Ma, Chapter 6, Lemma 4] that $\mathfrak{q} \in \operatorname{Ass}\left(M / g_{d} M\right)$, and so one can use Grothendieck's Finiteness Theorem (see [B-S, 9.5.2]) to see that $f_{R_{+}}\left(M / g_{d} M\right) \leq$ $0+$ ht $\left(\left(\mathfrak{q}+R_{+}\right) / \mathfrak{q}\right)=f-1$.

In the second case, when $\mathfrak{p} \notin \operatorname{Ass}_{R} M$, we choose $g_{d}$ as follows. First note that, for each $\mathfrak{q}^{\prime} \in \operatorname{Ass}_{R} M$, we have $\mathfrak{p} \cap R_{+} \not \subset \mathfrak{q}^{\prime}$. To see this, suppose that $\mathfrak{p} \cap R_{+} \subseteq \mathfrak{q}^{\prime}$ for some $\mathfrak{q}^{\prime} \in \operatorname{Ass}_{R} M$. Then $\mathfrak{p} \subset \mathfrak{q}^{\prime}$ (since $\Gamma_{R_{+}}(M)=0$ ), so that, since $\mathfrak{q}^{\prime} \cap R_{0} \supseteq \mathfrak{p} \cap R_{0}=\mathfrak{m}_{0}$, we have

$$
\operatorname{ht}\left(\left(\mathfrak{q}^{\prime}+R_{+}\right) / \mathfrak{q}^{\prime}\right)=\operatorname{ht}\left(\mathfrak{m} / \mathfrak{q}^{\prime}\right)<\operatorname{ht}(\mathfrak{m} / \mathfrak{p})=\operatorname{ht}\left(\left(\mathfrak{p}+R_{+}\right) / \mathfrak{p}\right) .
$$

This implies that depth $M_{\mathfrak{q}^{\prime}}+$ ht $\left(\left(\mathfrak{q}^{\prime}+R_{+}\right) / \mathfrak{q}^{\prime}\right)<f-1$, contrary to Grothendieck's Finiteness Theorem. We have therefore shown that $\mathfrak{p} \cap R_{+} \not \subset \mathfrak{q}^{\prime}$. As this is true for all $\mathfrak{q}^{\prime} \in \operatorname{Ass}_{R} M$, we can now use [B-S, 15.1.2] to see that there exist a positive integer $d$ and a homogeneous element $g_{d} \in \mathfrak{p} \cap R_{d}$ which is a non-zerodivisor on $M$. Note that $\operatorname{depth}\left(M / g_{d} M\right)_{\mathfrak{p}}=\operatorname{depth} M_{\mathfrak{p}}-1$, so that, by Grothendieck's Finiteness Theorem,

$$
f_{R_{+}}\left(M / g_{d} M\right) \leq \operatorname{depth}\left(M / g_{d} M\right)_{\mathfrak{p}}+\mathrm{ht}\left(\left(\mathfrak{p}+R_{+}\right) / \mathfrak{p}\right)=f-1 .
$$

Thus, in both cases, we have found a homogeneous element $g_{d}$ of $R$ of positive degree $d$ which is a non-zerodivisor on $M$ and is such that $f_{R_{+}}\left(M / g_{d} M\right) \leq f-1$. Application of local cohomology with respect to $R_{+}$to the exact sequence

$$
0 \longrightarrow M(-d) \stackrel{g_{d}}{\longrightarrow} M \longrightarrow M / g_{d} M \longrightarrow 0
$$

shows that $f_{R_{+}}\left(M / g_{d} M\right) \geq f-1$, and that, for all $n<<0$, the $R_{0}$-module $H_{R_{+}}^{f}(M)_{n}$ has a submodule isomorphic to $H_{R_{+}}^{f-1}\left(M / g_{d} M\right)_{n+d}$. It therefore follows that $f_{R_{+}}\left(M / g_{d} M\right)=f-1$ (so that $M / g_{d} M$ is not $R_{+}$-torsion), and we can apply the inductive hypothesis to $M / g_{d} M$.

In our first case, when $\mathfrak{p} \in \operatorname{Ass}_{R} M$, we have already noted that

$$
\mathfrak{q} \in \operatorname{Proj}(R) \cap \operatorname{Ass}\left(M / g_{d} M\right),
$$


that $\mathfrak{q} \cap R_{0}=\mathfrak{m}_{0}$, and that $\operatorname{depth}\left(M / g_{d} M\right)_{\mathfrak{q}}+\mathrm{ht}\left(\left(\mathfrak{q}+R_{+}\right) / \mathfrak{q}\right)=f-1$. We therefore use $\mathfrak{q}$ to draw a conclusion from the inductive hypothesis.

In our second case, when $\mathfrak{p} \notin \operatorname{Ass}_{R} M$, we noted that

$$
\operatorname{depth}\left(M / g_{d} M\right)_{\mathfrak{p}}+\operatorname{ht}\left(\left(\mathfrak{p}+R_{+}\right) / \mathfrak{p}\right)=f-1 ;
$$

in this case, we use $\mathfrak{p}$ to draw a conclusion from the inductive hypothesis.

In both cases, the inductive hypothesis yields that

$$
\mathfrak{m}_{0} \in \operatorname{Ass}_{R_{0}}\left(H_{R_{+}}^{f-1}\left(M / g_{d} M\right)_{n+d}\right)
$$

for all $n<<0$. Therefore $\mathfrak{m}_{0} \in \operatorname{Ass}_{R_{0}}\left(H_{R_{+}}^{f}(M)_{n}\right)$ for all $n<<0$, and the inductive step is complete.

We have thus proved that

$$
\operatorname{Ass}_{R_{0}}\left(H_{R_{+}}^{f}(M)_{n}\right) \supseteq\left\{\mathfrak{p} \cap R_{0}: \mathfrak{p} \in \operatorname{Proj}(R) \text { and } \operatorname{depth} M_{\mathfrak{p}}+\mathrm{ht}\left(\mathfrak{p}+R_{+}\right) / \mathfrak{p}=f\right\}
$$

for all $n<<0$.

To complete the proof, we suppose that $\mathfrak{p}_{0} \in \operatorname{Ass}_{R_{0}}\left(H_{R_{+}}^{f}(M)_{n}\right)$ for all $n<<0$; it is enough for us to show that there exists $\mathfrak{p} \in \operatorname{Proj}(R)$ with $\mathfrak{p} \cap R_{0}=\mathfrak{p}_{0}$ and $\operatorname{depth} M_{\mathfrak{p}}+\mathrm{ht}\left(\mathfrak{p}+R_{+}\right) / \mathfrak{p}=f$. Our first steps in this direction show that additional simplifications are posssible.

Invert $R_{0} \backslash \mathfrak{p}_{0}$; in other words, apply homogeneous localization at $\mathfrak{p}_{0}+R_{+}$. Observe that the hypotheses imply that $\left(R_{0}\right)_{\mathfrak{p}_{0}}$ is a homomorphic image of a regular local ring $\left(R_{0}^{\prime}, \mathfrak{m}_{0}^{\prime}\right)$ and that $R_{\left(\mathfrak{p}_{0}+R_{+}\right)}$is an image of a polynomial ring $R^{\prime}:=R_{0}^{\prime}\left[X_{1}, \ldots, X_{r}\right]$, graded in the usual way, under a ring homomorphism which is homogeneous in the sense of [B-S, Definition 13.1.2]. Consider $M$ as a finitely generated graded $R^{\prime}$-module; we can then use the Graded Independence Theorem [B-S, 13.1.6] to see that $f=f_{R_{+}^{\prime}}(M)$ and that it is enough for us establish the existence of a $\mathfrak{p} \in \operatorname{Proj}(R)$ with the specified properties under the additional hypotheses that $\left(R_{0}, \mathfrak{m}_{0}\right)$ is a regular local ring, that $\mathfrak{p}_{0}=\mathfrak{m}_{0}$, and that $R=R_{0}\left[X_{1}, \ldots, X_{r}\right]$, a polynomial ring graded in the usual way.

We deal first with the case where $r=1$. Then $f=1$. Set $\bar{M}:=M / \Gamma_{R_{+}}(M)$, and recall that there is a homogeneous isomorphism $H_{R_{+}}^{1}(M) \stackrel{\cong}{\longrightarrow} H_{R_{+}}^{1}(\bar{M})$. Therefore, since $M_{\mathfrak{q}} \cong \bar{M}_{\mathfrak{q}}$ for all $\mathfrak{q} \in \operatorname{Spec}(R) \backslash \operatorname{Var}\left(R_{+}\right)$, we can, and do, impose the additional hypothesis that $\Gamma_{R_{+}}(M)=0$. (Here, $\operatorname{Var}(\mathfrak{a})$, for an ideal $\mathfrak{a}$ of $R$, denotes the variety of a.)

By hypothesis, $\left(0:_{H_{R_{+}}^{1}}(M): \mathfrak{m}_{0}\right)_{n} \neq 0$ for all $n<<$. It therefore follows that the graded $R$-module $\Gamma_{\mathfrak{m}_{0} R}\left(H_{R_{+}}^{1}(M)\right)$ is not finitely generated.

Let

$$
0 \longrightarrow{ }^{*} E^{0}(M) \stackrel{d^{0}}{\longrightarrow} * E^{1}(M) \stackrel{d^{1}}{\longrightarrow} * E^{2}(M) \longrightarrow \cdots \longrightarrow{ }^{*} E^{i}(M) \longrightarrow \cdots
$$

be the minimal $*$ injective resolution of $M$, with associated (necessarily homogeneous) augmentation homomorphism $d^{-1}: M \longrightarrow{ }^{*} E^{0}(M)$. Since $\Gamma_{R_{+}}(M)=0$, it follows from [S, Theorem 2.4] that $\Gamma_{R_{+}}\left({ }^{*} E^{0}(M)\right)=0$, so that $\Gamma_{\mathfrak{m}}\left({ }^{*} E^{0}(M)\right)=0$. Therefore

$$
H_{R_{+}}^{1}(M) \cong \operatorname{Ker}\left(\Gamma_{R_{+}}\left(d^{1}\right)\right) \quad \text { and } \quad H_{\mathfrak{m}}^{1}(M) \cong \operatorname{Ker}\left(\Gamma_{\mathfrak{m}}\left(d^{1}\right)\right) .
$$


Here, $\Gamma_{R_{+}}\left(d^{1}\right): \Gamma_{R_{+}}\left({ }^{*} E^{1}(M)\right) \longrightarrow \Gamma_{R_{+}}\left({ }^{*} E^{2}(M)\right)$ is the map induced by $d^{1}$, et cetera. Thus

$$
\Gamma_{\mathfrak{m}_{0} R}\left(H_{R_{+}}^{1}(M)\right) \cong \Gamma_{\mathfrak{m}_{0} R}\left(\operatorname{Ker}\left(\Gamma_{R_{+}}\left(d^{1}\right)\right)\right)=\operatorname{Ker}\left(\Gamma_{\mathfrak{m}}\left(d^{1}\right)\right) \cong H_{\mathfrak{m}}^{1}(M) .
$$

Therefore, $H_{\mathfrak{m}}^{1}(M)$ is not finitely generated. Hence, by Grothendieck's Finiteness Theorem (see [B-S, 13.1.17]), there exists $\mathfrak{p} \in{ }^{*} \operatorname{Spec}(R) \backslash \operatorname{Var}(\mathfrak{m})$ such that $\operatorname{depth} M_{\mathfrak{p}}+\mathrm{ht} \mathfrak{m} / \mathfrak{p}=1$. This means that $\mathfrak{p} \in \operatorname{Ass}_{R} M$ and $h t \mathfrak{m} / \mathfrak{p}=1$. Note that $\mathfrak{p} \nsupseteq R_{+}$, because $\Gamma_{R_{+}}(M)=0$. Therefore $\mathfrak{p}_{0}:=\mathfrak{p} \cap R_{0}=\mathfrak{m}_{0}$, since otherwise $\mathfrak{m} \supset \mathfrak{p}_{0}+R_{+} \supset \mathfrak{p}$ would be a chain of distinct prime ideals of $R$, contrary to the fact that ht $\mathfrak{m} / \mathfrak{p}=1$. The claim is therefore proved in the case where $r=1$.

Now suppose that $r \geq 2$, and that the desired result has been proved for smaller values of $r$. Note that, by Grothendieck's Finiteness Theorem, it is enough for us to show that there exists $\mathfrak{p} \in \operatorname{Proj}(R) \cap \operatorname{Var}\left(\mathfrak{m}_{0} R\right)$ with depth $M_{\mathfrak{p}}+\mathrm{ht}\left(\mathfrak{p}+R_{+}\right) / \mathfrak{p} \leq f$.

By Lemma 1.7, there exists a regular local flat extension ring $\left(R_{0}^{\prime}, \mathfrak{m}_{0}^{\prime}\right)$ of $R_{0}$ such that $\mathfrak{m}_{0} R_{0}^{\prime}=\mathfrak{m}_{0}^{\prime}$ and $R_{0}^{\prime} / \mathfrak{m}_{0}^{\prime}$ is algebraically closed. Let $R^{\prime}=R \otimes_{R_{0}} R_{0}^{\prime}$, which we identify with $R_{0}^{\prime}\left[X_{1}, \ldots, X_{r}\right]$ in the obvious way. Let $M^{\prime}$ denote the finitely generated graded $R^{\prime}$-module $M \otimes_{R} R^{\prime}$. It follows from [B-S, 13.1.8 and 15.2.2] that $f_{R_{+}^{\prime}}\left(M^{\prime}\right)=f$ and $\mathfrak{m}_{0}^{\prime} \in \operatorname{Ass}_{R_{0}^{\prime}}\left(H_{R_{+}^{\prime}}^{f}\left(M^{\prime}\right)_{n}\right)$ for all $n<<0$.

Suppose that we have found $\mathfrak{p}^{\prime} \in \operatorname{Proj}\left(R^{\prime}\right) \cap \operatorname{Var}\left(\mathfrak{m}_{0}^{\prime} R^{\prime}\right)$ such that

$$
\operatorname{depth} M_{\mathfrak{p}^{\prime}}^{\prime}+\operatorname{ht}\left(\mathfrak{p}^{\prime}+R_{+}^{\prime}\right) / \mathfrak{p}^{\prime} \leq f .
$$

Set $\mathfrak{p}:=\mathfrak{p}^{\prime} \cap R$. Then it follows from Lemma 1.4 that $\mathfrak{p} \in \operatorname{Proj}(R) \cap \operatorname{Var}\left(\mathfrak{m}_{0} R\right)$ and

$$
\operatorname{depth} M_{\mathfrak{p}}+\operatorname{ht}\left(\mathfrak{p}+R_{+}\right) / \mathfrak{p} \leq \operatorname{depth}_{R_{\mathfrak{p}^{\prime}}^{\prime}} M_{\mathfrak{p}^{\prime}}^{\prime}+\operatorname{ht}\left(\mathfrak{p}^{\prime}+R_{+}^{\prime}\right) / \mathfrak{p}^{\prime} \leq f .
$$

Therefore we can, and do, assume for the remainder of this proof that $R_{0} / \mathfrak{m}_{0}$ is algebraically closed.

We now proceed by descending induction on $f$. Note that $f \leq r$. So we deal first with the case where $f=r$. By Lemma 1.5, there exists $y_{r} \in R_{1} \backslash \mathfrak{m}_{0} R_{1}$ such that $y_{r}$ is a non-zerodivisor on $M / \Gamma_{R_{+}}(M)$, that $f_{R_{+}}\left(M / y_{r} M\right)=r-1$ and that

$$
\mathfrak{m}_{0} \in \operatorname{Ass}_{R_{0}}\left(H_{R_{+}}^{r-1}\left(M / y_{r} M\right)_{n}\right) \quad \text { for all } n<<0 .
$$

Since the image of $y_{r}$ in $R_{1} / \mathfrak{m}_{0} R_{1}$ is non-zero, there exist $y_{1}, \ldots, y_{r-1} \in R_{1}$ such that $R_{1}$ is generated (over $R_{0}$ ) by $y_{1}, \ldots, y_{r-1}, y_{r}$. Note that

$$
R=R_{0}\left[y_{1}, \ldots, y_{r-1}, y_{r}\right]
$$

and that $y_{1}, \ldots, y_{r-1}, y_{r}$ are algebraically independent over $R_{0}$. Therefore, we can, and do, assume that $y_{r}=X_{r}$.

We can consider $M / X_{r} M$ as a finitely generated graded module over $R / X_{r} R$. The Graded Independence Theorem [B-S 13.1.6] shows that $f_{\left(R / X_{r} R\right)_{+}}\left(M / X_{r} M\right)=$ $r-1$ and that

$$
\mathfrak{m}_{0} \in \operatorname{Ass}_{R_{0}}\left(H_{\left(R / X_{r} R\right)_{+}}^{r-1}\left(M / X_{r} M\right)_{n}\right) \quad \text { for all } n<<0 .
$$

Since $R / X_{r} R$ is (homogeneously) isomorphic to $R_{0}\left[X_{1}, \ldots, X_{r-1}\right]$, we may apply the inductive hypothesis to deduce that there exists

$$
\overline{\mathfrak{p}} \in \operatorname{Proj}\left(R / X_{r} R\right) \cap \operatorname{Var}\left(\mathfrak{m}_{0}\left(R / X_{r} R\right)\right)
$$

with

$$
\operatorname{depth}\left(M / X_{r} M\right)_{\overline{\mathfrak{p}}}+\operatorname{ht}\left(\overline{\mathfrak{p}}+\left(R / X_{r} R\right)_{+}\right) / \overline{\mathfrak{p}} \leq r-1 .
$$


Let $\mathfrak{p}$ be the inverse image of $\overline{\mathfrak{p}}$ under the natural ring homomorphism $R \rightarrow R / X_{r} R$. Then $X_{r} \in \mathfrak{p}$ and $\mathfrak{p} \in \operatorname{Proj}(R) \cap \operatorname{Var}\left(\mathfrak{m}_{0} R\right) ;$ also depth $M_{\mathfrak{p}}=\operatorname{depth}\left(M / X_{r} M\right)_{\bar{p}}+1$ (because $\left.M_{\mathfrak{p}} \cong\left(M / \Gamma_{R_{+}}(M)\right)_{\mathfrak{p}}\right)$ and

$$
\operatorname{ht}\left(\mathfrak{p}+R_{+}\right) / \mathfrak{p}=\operatorname{ht}\left(\overline{\mathfrak{p}}+\left(R / X_{r} R\right)_{+}\right) / \overline{\mathfrak{p}}
$$

Hence depth $M_{\mathfrak{p}}+\mathrm{ht}\left(\mathfrak{p}+R_{+}\right) / \mathfrak{p} \leq r$. Thus we have found a $\mathfrak{p}$ with the required properties in the case where $f=r>1$.

Now suppose that $f<r$ and that the desired result has been proved for larger values of $f$ (for this value of $r$ ). There is an exact sequence $0 \rightarrow N \rightarrow F \rightarrow M \rightarrow 0$ of finitely generated graded $R$-modules and homogeneous homomorphisms in which $F$ is free. By Lemma 1.6(iii), we have $f_{R_{+}}(N)=f+1$; by part (ii) of the same lemma, $\mathfrak{m}_{0} \in \operatorname{Ass}_{R_{0}}\left(H_{R_{+}}^{f+1}(N)_{n}\right)$ for all $n<<0$. Therefore, by the inductive hypothesis, there exists $\mathfrak{p} \in \operatorname{Proj}(R) \cap \operatorname{Var}\left(\mathfrak{m}_{0} R\right)$ with depth $N_{\mathfrak{p}}+\operatorname{ht}\left(\mathfrak{p}+R_{+}\right) / \mathfrak{p} \leq f+1$.

Note that $\operatorname{depth} M_{\mathfrak{p}} \leq \mathrm{ht} \mathfrak{p}$ : we consider the cases where $\operatorname{depth} M_{\mathfrak{p}}<$ ht $\mathfrak{p}$ and $\operatorname{depth} M_{\mathfrak{p}}=$ ht $\mathfrak{p}$ separately. When depth $M_{\mathfrak{p}}<$ ht $\mathfrak{p}$, it follows from Lemma 1.6 (i) that depth $N_{\mathfrak{p}}=\operatorname{depth} M_{\mathfrak{p}}+1$; therefore depth $M_{\mathfrak{p}}+\mathrm{ht}\left(\mathfrak{p}+R_{+}\right) / \mathfrak{p} \leq f$. In the other case, depth $M_{\mathfrak{p}}=$ ht $\mathfrak{p}$, so that, again by Lemma 1.6(i), depth $N_{\mathfrak{p}}=$ ht $\mathfrak{p}$. Therefore, in this case,

$$
\begin{aligned}
\operatorname{ht}\left(\mathfrak{m}_{0}+R_{+}\right) & =\operatorname{ht}\left(\mathfrak{p}+R_{+}\right)=\operatorname{ht}\left(\mathfrak{p}+R_{+}\right) / \mathfrak{p}+\mathrm{ht} \mathfrak{p} \\
& =\operatorname{ht}\left(\mathfrak{p}+R_{+}\right) / \mathfrak{p}+\operatorname{depth} N_{\mathfrak{p}} \leq f+1 \leq r .
\end{aligned}
$$

Therefore $\mathfrak{m}_{0}=0$, and $R_{0}$ is a field. In this case, the desired conclusion is clear from the graded version of Grothendieck's Finiteness Theorem (see [B-S, 13.1.17]). The proof is now complete.

\section{Further examination of Singh's example}

In [Si, §4], A. K. Singh showed that the ring

$$
R^{\prime}:=\mathbb{Z}[X, Y, Z, U, V, W] /(X U+Y V+Z W),
$$

where $X, Y, Z, U, V, W$ are independent indeterminates over $\mathbb{Z}$, has the property that $\operatorname{Ass}_{R^{\prime}}\left(H_{\mathfrak{a}}^{3}\left(R^{\prime}\right)\right)$ is infinite, where $\mathfrak{a}$ is the ideal generated by the images of $U, V, W$. Brodmann and Hellus [B-H], (5.7)(A)] observed that Singh's argument leads to an interesting conclusion about graded components of graded local cohomology modules: we can consider $\mathbb{Z}[X, Y, Z, U, V, W]$ as a positively graded ring with 0 th component $\mathbb{Z}[X, Y, Z]$ and $U, V, W$ each assigned degree $1 ; R^{\prime}$ inherits a structure as a standard positively graded ring with $R_{+}^{\prime}=\mathfrak{a}$; the argument Singh used to prove his result mentioned above actually shows that $\left\{\mathfrak{p} \cap \mathbb{Z}: \mathfrak{p} \in \operatorname{Ass}_{R^{\prime}}\left(H_{R_{+}^{\prime}}^{3}\left(R^{\prime}\right)\right)\right\}$ is an infinite set, and Brodmann and Hellus noted that this implies that $\operatorname{Ass}_{R_{0}^{\prime}}\left(H_{R_{+}^{\prime}}^{3}\left(R^{\prime}\right)_{n}\right)$ is not asymptotically stable for $n \longrightarrow \infty$.

Our aim in the rest of this paper is to use Gröbner basis techniques on Singh's example to identify precisely the set $\operatorname{Ass}_{R_{0}^{\prime}}\left(H_{R_{+}^{\prime}}^{3}\left(R^{\prime}\right)_{n}\right)$ for each $n \leq-3$, and to then deduce that $\operatorname{Ass}_{R_{0}^{\prime}}\left(H_{R_{+}^{\prime}}^{3}\left(R^{\prime}\right)_{n}\right)$ is not asymptotically increasing for $n \longrightarrow \infty$.

2.1. Notation. Throughout the rest of the paper, the symbol $L$ will denote either a field or a principal ideal domain (PID), and $R$ will denote the polynomial ring $L[X, Y, Z, U, V, W]$, graded so that $U, V, W$ have degree 1 and $X, Y, Z$ have degree 0 ; thus $R_{0}=L[X, Y, Z]$. We shall set $F:=X U+Y V+Z W$, and $R^{\prime}:=R / F R$, again a standard positively graded ring. The natural map $R \rightarrow R^{\prime}$ maps $R_{0}$ isomorphically 
onto $R_{0}^{\prime}$, and so we shall identify elements of $R_{0}$ with their natural images in $R_{0}^{\prime}$. In the case where $L=\mathbb{Z}$, the rings $R$ and $R^{\prime}$ are those occurring in Singh's example mentioned above. However, it will be helpful in another context to have some calculations available in the case where $L$ is the rational field, for example.

Since $H_{R_{+}^{\prime}}^{3}\left(R^{\prime}\right)$ is homogeneously isomorphic to $H_{R_{+}}^{3}(R / F R)$, we can use the exact sequence

$$
H_{R_{+}}^{3}(R)(-1) \stackrel{F}{\longrightarrow} H_{R_{+}}^{3}(R) \longrightarrow H_{R_{+}}^{3}(R / F R) \longrightarrow 0
$$

of graded $R$-modules and homogeneous homomorphisms (induced from the exact sequence

$$
0 \longrightarrow R(-1) \stackrel{F}{\longrightarrow} R \longrightarrow R / F R \longrightarrow 0)
$$

to study $H_{R_{+}^{\prime}}^{3}\left(R^{\prime}\right)$. Also, we can realize $H_{R_{+}}^{3}(R)$ as the module $R_{0}\left[U^{-}, V^{-}, W^{-}\right]$of inverse polynomials described in $[\mathrm{B}-\mathrm{S}, 12.4 .1]$ : this graded $R$-module has end -3 , and, for each $d \geq 3$, its $(-d)$-th component is a free $R_{0}$-module of rank $\left(\begin{array}{c}d-1 \\ 2\end{array}\right)$ with base $\left(U^{\alpha} V^{\beta} W^{\gamma}\right)_{-\alpha,-\beta,-\gamma \in \mathbb{N}, \alpha+\beta+\gamma=-d}$. We plan to study the graded components of $H_{R_{+}}^{3}(R / F R)$ by considering the cokernels of the $R_{0}$-homomorphisms

$$
F_{-d}: R_{0}\left[U^{-}, V^{-}, W^{-}\right]_{-d-1} \longrightarrow R_{0}\left[U^{-}, V^{-}, W^{-}\right]_{-d} \quad(d \geq 3)
$$

given by multiplication by $F$. In order to represent these $R_{0}$-homomorphisms between free $R_{0}$-modules by matrices, we specify an ordering for each of the abovementioned bases by declaring that

$$
U^{\alpha_{1}} V^{\beta_{1}} W^{\gamma_{1}}<U^{\alpha_{2}} V^{\beta_{2}} W^{\gamma_{2}}
$$

(where $-\alpha_{i},-\beta_{i},-\gamma_{i} \in \mathbb{N}$ and $\alpha_{i}+\beta_{i}+\gamma_{i}=n \leq-3$ for $i=1,2$ ) precisely when $\alpha_{1}>\alpha_{2}$ or $\alpha_{1}=\alpha_{2}$ and $\beta_{1}>\beta_{2}$. For example, this ordering on our base for $R_{0}\left[U^{-}, V^{-}, W^{-}\right]_{-5}$ is such that

$$
\begin{aligned}
U^{-1} V^{-1} W^{-3} & <U^{-1} V^{-2} W^{-2}<U^{-1} V^{-3} W^{-1}<U^{-2} V^{-1} W^{-2} \\
& <U^{-2} V^{-2} W^{-1}<U^{-3} V^{-1} W^{-1} .
\end{aligned}
$$

We shall frequently need to consider an $R_{0}$-homomorphism from the free $R_{0^{-}}$ module $R_{0}^{n}$ (regarded as consisting of column vectors) to $R_{0}^{m}$ (where $m$ and $n$ are positive integers) given by left multiplication by an $m \times n$ matrix $C$ with entries in $R_{0}$. In these circumstances, we shall also use $C$ to denote the homomorphism; its image $\operatorname{Im} C$ is just the submodule of $R_{0}^{m}$ generated by the columns of $C$, for if $\left(\mathbf{e}_{i}\right)_{i=1, \ldots, n}$ denotes the standard base for $R_{0}^{n}$, then $C \mathbf{e}_{j}$ is just the $j$-th column of $C$ (for $1 \leq j \leq n)$.

The theory of Gröbner bases is well developed for ideals in polynomial rings in finitely many indeterminates with coefficients in a principal ideal domain, and for submodules of finite free modules over polynomial rings in finitely many indeterminates over a field (see, for example, [A-L, Chapter 3]). It is straightforward to combine the methods from these two parts of the theory to produce a theory of Gröbner bases for submodules of finite free $L[X, Y, Z]$-modules. Thus much of the work below applies both to the case where $L$ is a PID and the case where $L$ is a field.

In this paper, we use the lexicographical term order with $X>Y>Z$ in $R_{0}$, and for each $n \in \mathbb{N}$ we set $>$ to be the 'term-over-position' extension of this order to $R_{0}^{n}$ defined as follows: a monomial in $R_{0}^{n}$ is a column vector of the form $m \mathbf{e}_{j}$, where $m$ is a monomial in $R_{0}$ and $\mathbf{e}_{j}$ is the $j$-th standard base vector of $R_{0}^{n}$; and 
$m_{1} \mathbf{e}_{j_{1}}>m_{2} \mathbf{e}_{j_{2}}$ (for monomials $m_{1}, m_{2}$ of $R_{0}$ and $j_{1}, j_{2} \in\{1, \ldots, n\}$ ) if and only if $m_{1}>m_{2} \quad$ or $\quad m_{1}=m_{2}$ and $j_{1}<j_{2}$.

If $A$ is an $m \times n$ matrix with entries in $R_{0}$ (we shall say 'over $R_{0}$ ') and $\mathbf{f}, \mathbf{h} \in R_{0}^{n}$, then we shall say that $\mathbf{f}$ reduces to $\mathbf{h}$ modulo $A$, denoted by $\mathbf{f} \stackrel{A}{\longrightarrow}+\mathbf{h}$, when $\mathbf{f}$ reduces to $\mathbf{h}$ modulo the set of columns of $A$ (see [A-L, Definition 3.5.8], but modify that definition to imitate [A-L, Definitions 4.1.1 and 4.1.6] in the case where $L$ is a PID).

We shall denote the leading monomial, leading coefficient and leading term of $\mathbf{f} \in R_{0}^{n}$ by $\operatorname{lm}(\mathbf{f}), \operatorname{lc}(\mathbf{f})$ and $\operatorname{lt}(\mathbf{f})$ respectively.

We shall use $I_{n}$ to denote the $n \times n$ identity matrix. For each $n \in \mathbb{N}$, we let $A_{n}$ denote the $n \times(n+1)$ matrix given by

$$
A_{n}=\left[\begin{array}{ccccc}
Z & Y & 0 & \ldots & 0 \\
0 & Z & Y & 0 & \cdots \\
\vdots & & \ddots & \ddots & \\
0 & \cdots & 0 & Z & Y
\end{array}\right]
$$

2.2. Lemma. Let $d \in \mathbb{N}$ with $d \geq 3$.

(i) With the notation of 2.1, the $R_{0}$-homomorphism

$$
F_{-d}: R_{0}\left[U^{-}, V^{-}, W^{-}\right]_{-d-1} \longrightarrow R_{0}\left[U^{-}, V^{-}, W^{-}\right]_{-d}
$$

given by multiplication by $F$ is represented, relative to the bases specified in 2.1 listed in increasing order, by the $\left(\begin{array}{c}d-1 \\ 2\end{array}\right) \times\left(\begin{array}{l}d \\ 2\end{array}\right)$ matrix

$$
T_{d}:=\left[\begin{array}{cccccc}
A_{d-2} & X I_{d-2} & 0 & \ldots & & 0 \\
0 & A_{d-3} & X I_{d-3} & 0 & \ldots & 0 \\
0 & 0 & A_{d-4} & X I_{d-4} & & 0 \\
\vdots & \vdots & & \ddots & \ddots & \vdots \\
0 & 0 & \ldots & 0 & A_{1} & X I_{1}
\end{array}\right],
$$

where $A_{d-2}, \ldots, A_{1}$ are as defined in 2.1.

(ii) Each associated prime in $\operatorname{Ass}_{R_{0}^{\prime}}\left(H_{R_{+}^{\prime}}^{3}\left(R^{\prime}\right)_{-d}\right)$ contains $X, Y$ and $Z$.

(iii) $(X, Y, Z) \in \operatorname{Ass}_{R_{0}^{\prime}}\left(H_{R_{+}^{\prime}}^{3}\left(R^{\prime}\right)_{-d}\right)$.

Proof. (i) This follows from the fact that, for negative integers $\alpha, \beta, \gamma$,

$$
\begin{aligned}
F\left(U^{\alpha} V^{\beta} W^{\gamma}\right)= & X\left(1-\delta_{\alpha,-1}\right) U^{\alpha+1} V^{\beta} W^{\gamma}+Y\left(1-\delta_{\beta,-1}\right) U^{\alpha} V^{\beta+1} W^{\gamma} \\
& +Z\left(1-\delta_{\gamma,-1}\right) U^{\alpha} V^{\beta} W^{\gamma+1},
\end{aligned}
$$

where $\delta_{i, j}$ is Kronecker's delta.

(ii) Consider the last column of $T_{d}$ to see that $X \mathbf{e}_{\left(\begin{array}{c}d-1 \\ 2\end{array}\right)} \in \operatorname{Im} T_{d}$; therefore $X Y \mathbf{e}_{\left(\begin{array}{c}d-1 \\ 2\end{array}\right)}, X Z \mathbf{e}_{\left(\begin{array}{c}d-1 \\ 2\end{array}\right)} \in \operatorname{Im} T_{d}$, so that $X^{2} \mathbf{e}_{\left(\begin{array}{c}d-1 \\ 2\end{array}\right)-1}, X^{2} \mathbf{e}_{\left(\begin{array}{c}d-1 \\ 2\end{array}\right)-2} \in \operatorname{Im} T_{d}$ in view of the next-to-last and second-to-last columns of $T_{d}$; we can now continue in this way to see that each element of Coker $T_{d}=$ Coker $F_{-d}$ is annihilated by $X^{d-2}$. By symmetry, $Y^{d-2}$ and $Z^{d-2}$ also annihilate Coker $F_{-d}=$ Coker $T_{d}$.

(iii) It is clear from part (i) that $\left(\operatorname{Im} F_{-3}:_{R_{0}} U^{-1} V^{-1} W^{-1}\right)=(X, Y, Z)$. Hence $\left(0: R_{0}\right.$ Coker $\left.F_{-3}\right)=(X, Y, Z)$. Now multiplication by $U^{d-3}$ induces an $R_{0^{-}}$ epimorphism Coker $F_{-d} \longrightarrow$ Coker $F_{-3}$, so that, in view of the above proof of part (ii), we have

$$
\left(X^{d-2}, Y^{d-2}, Z^{d-2}\right) \subseteq\left(0:_{R_{0}} \text { Coker } F_{-d}\right) \subseteq\left(0:_{R_{0}} \text { Coker } F_{-3}\right)=(X, Y, Z) .
$$


Therefore $(X, Y, Z)$ is a minimal member of the support of Coker $F_{-d}$.

2.3. Lemma. Let $k, m, n, q \in \mathbb{N}_{0}$ with $m, n>0$. Let $A=\left[a_{i j}\right]$ be an $m \times n$ matrix with entries in $L[Y, Z]$, let $\mathbf{f} \in L[Y, Z]^{n}$, and let $M$ and $M^{\prime}$ denote the $(k+n+m+q)$-rowed block matrices over $R_{0}$ given by

$$
M:=\left[\begin{array}{cc}
0 & 0 \\
X I_{n} & \mathbf{f} \\
A & 0 \\
0 & 0
\end{array}\right] \quad \text { and } \quad M^{\prime}:=\left[\begin{array}{c}
0 \\
X I_{n} \\
A \\
0
\end{array}\right]
$$

in which the first $k$ and last $q$ rows are all zero.

Then each S-polynomial of two columns of $M$ is either 0 or reduces modulo $M^{\prime}$ to

$$
\left[\begin{array}{c}
0 \\
0 \\
\pm A \mathbf{f} \\
0
\end{array}\right]
$$

(in which the lowest ' 0 ' stands for the $q \times 1$ zero matrix), and these column matrices do arise from $S$-polynomials in this way.

Proof. Suppose $\mathbf{f} \neq 0$, and let $\mathbf{f}=\sum_{j=1}^{t} c_{i_{j}} T_{i_{j}} \mathbf{e}_{i_{j}}$ be an expression for $\mathbf{f}$ as a sum of terms, where the $T_{i_{j}}$ are monomials in $Y$ and $Z$ and the $c_{i_{j}}$ are elements of $L$; suppose that $\operatorname{lt}(\mathbf{f})=c_{i_{h}} T_{i_{h}} \mathbf{e}_{i_{h}}$. Let $\mathbf{m}_{j}$ denote the $j$ th column of $M$, for each $j=1, \ldots, n+1$.

Since $\mathbf{f} \in L[Y, Z]$, we have $\operatorname{lcm}\left(T_{i_{h}}, X\right)=T_{i_{h}} X$. All $S$-polynomials of two columns of $M$ are zero except possibly for those of $\mathbf{m}_{i_{h}}$ and $\mathbf{m}_{n+1}$. Note that

$$
\mathbf{m}_{n+1}=\sum_{j=1}^{t} c_{i_{j}} T_{i_{j}} \mathbf{e}_{i_{j}+k} \quad \text { and } \quad \mathbf{m}_{i}=X \mathbf{e}_{i+k}+\sum_{\rho=1}^{m} a_{\rho i} \mathbf{e}_{\rho+k+n}(1 \leq i \leq n) .
$$

We have

$$
\begin{aligned}
S\left(\mathbf{m}_{i_{h}}, \mathbf{m}_{n+1}\right) & =\frac{c_{i_{h}}}{1} \frac{T_{i_{h}} X}{X} \mathbf{m}_{i_{h}}-\frac{c_{i_{h}}}{c_{i_{h}}} \frac{T_{i_{h}} X}{T_{i_{h}}} \mathbf{m}_{n+1}=c_{i_{h}} T_{i_{h}} \mathbf{m}_{i_{h}}-X \mathbf{m}_{n+1} \\
& =c_{i_{h}} T_{i_{h}} X \mathbf{e}_{i_{h}+k}+\sum_{\rho=1}^{m} a_{\rho i_{h}} c_{i_{h}} T_{i_{h}} \mathbf{e}_{\rho+k+n}-\sum_{j=1}^{t} c_{i_{j}} X T_{i_{j}} \mathbf{e}_{i_{j}+k} \\
& =\sum_{\rho=1}^{m} a_{\rho i_{h}} c_{i_{h}} T_{i_{h}} \mathbf{e}_{\rho+k+n}-\sum_{\substack{j=1 \\
j \neq h}}^{t} c_{i_{j}} X T_{i_{j}} \mathbf{e}_{i_{j}+k} \\
& \stackrel{M^{\prime}}{\longrightarrow}+\sum_{\rho=1}^{m} a_{\rho i_{h}} c_{i_{h}} T_{i_{h}} \mathbf{e}_{\rho+k+n}-\sum_{\substack{j=1 \\
j \neq h}}^{t} c_{i_{j}} X T_{i_{j}} \mathbf{e}_{i_{j}+k}+\sum_{\substack{j=1 \\
j \neq h}}^{t} c_{i_{j}} T_{i_{j}} \mathbf{m}_{i_{j}} \\
& =\sum_{j=1}^{t} \sum_{\rho=1}^{m} a_{\rho i_{j}} c_{i_{j}} T_{i_{j}} \mathbf{e}_{\rho+k+n}=\left[\begin{array}{c}
0 \\
0 \\
A \mathbf{f} \\
0
\end{array}\right],
\end{aligned}
$$

as claimed. 
2.4. Theorem. Consider the matrix

$$
T_{d}:=\left[\begin{array}{cccccc}
A_{d-2} & X I_{d-2} & 0 & \cdots & & 0 \\
0 & A_{d-3} & X I_{d-3} & 0 & \cdots & 0 \\
0 & 0 & A_{d-4} & X I_{d-4} & & 0 \\
\vdots & \vdots & & \ddots & \ddots & \vdots \\
0 & 0 & \cdots & 0 & A_{1} & X I_{1}
\end{array}\right]
$$

of 2.2. Define matrices $G_{d-2}, G_{d-3}, \ldots, G_{1}$ by descending induction as follows: let $G_{d-2}$ be a $(d-2)$-rowed matrix with entries in $L[Y, Z]$ whose columns include those of $A_{d-2}$ and provide a Gröbner basis for $\operatorname{Im} A_{d-2} ;$ for $i \in \mathbb{N}$ with $d-2>i \geq 1$, on the assumption that $G_{i+1}$ has been defined as an $(i+1)$-rowed matrix with entries in $L[Y, Z]$, let $G_{i}$ be an $i$-rowed matrix with entries in $L[Y, Z]$ whose columns include those of $A_{i} G_{i+1}$ and provide a Gröbner basis for $\operatorname{Im} A_{i} G_{i+1}$. Then:

(i) the columns of

$T_{d}^{\prime}:=\left[\begin{array}{cccccc|ccccc}A_{d-2} & X I_{d-2} & 0 & \ldots & & 0 & G_{d-2} & 0 & \ldots & & 0 \\ 0 & A_{d-3} & X I_{d-3} & 0 & \ldots & 0 & 0 & G_{d-3} & 0 & \ldots & 0 \\ 0 & 0 & A_{d-4} & X I_{d-4} & & 0 & 0 & 0 & G_{d-4} & & 0 \\ \vdots & \vdots & & \ddots & \ddots & \vdots & \vdots & \vdots & & \ddots & \vdots \\ 0 & 0 & \ldots & 0 & A_{1} & X I_{1} & 0 & 0 & \ldots & 0 & G_{1}\end{array}\right]$

form a Gröbner basis for $\operatorname{Im} T_{d}^{\prime}=\operatorname{Im} T_{d} ;$ and

(ii) the columns of

$$
H_{d}:=\left[\begin{array}{ccccc}
A_{d-2} & 0 & 0 & \cdots & 0 \\
0 & A_{d-3} A_{d-2} & 0 & \cdots & 0 \\
0 & 0 & A_{d-4} A_{d-3} A_{d-2} & \ldots & 0 \\
\vdots & \vdots & & \ddots & \vdots \\
0 & 0 & \ldots & & A_{1} A_{2} \ldots A_{d-2}
\end{array}\right]
$$

generate $\operatorname{Im} T_{d} \cap L[Y, Z]^{\left(\begin{array}{c}d-1 \\ 2\end{array}\right) .}$.

Proof. (i) Let $\mathbf{s}=S(\mathbf{f}, \mathbf{g})$ be a non-zero $S$-polynomial of two columns $\mathbf{f}$ and $\mathbf{g}$ of $T_{d}^{\prime}$. There are various cases to consider.

First of all, if $\mathbf{f}$ and $\mathbf{g}$ have leading terms in one of the first $d-2$ rows of $T_{d}^{\prime}$, then either $\mathbf{s} \stackrel{T_{d}^{\prime}}{\longrightarrow}+0$ because the columns of $\left[A_{d-2} \mid G_{d-2}\right]$ form a Gröbner basis, or else $\mathbf{s}$ reduces modulo $T_{d}^{\prime}$ to a column of

$$
\left[\begin{array}{c}
0 \\
\pm A_{d-3} G_{d-2} \\
0 \\
\vdots \\
0
\end{array}\right]
$$

by Lemma 2.3 since the columns of $G_{d-3}$ include the columns of $A_{d-3} G_{d-2}$, it follows that $\mathbf{s} \stackrel{T_{d}^{\prime}}{\longrightarrow}+0$ in this case also. 
Now suppose that $i \in \mathbb{N}$ with $d-2>i>1$ and that $\mathbf{f}$ and $\mathbf{g}$ have leading terms in row

$$
k+\sum_{j=i+1}^{d-2} j \quad \text { for some } k \in\{1, \ldots, i\} \text {. }
$$

Then either $\mathbf{s} \stackrel{T_{d}^{\prime}}{\longrightarrow}+0$ because the columns of $G_{i}$ form a Gröbner basis, or else $\mathbf{s}$ reduces modulo $T_{d}^{\prime}$ to a column of

$$
\left[\begin{array}{c}
0 \\
\vdots \\
0 \\
\pm A_{i-1} G_{i} \\
0 \\
\vdots \\
0
\end{array}\right]
$$

(where the block $A_{i-1} G_{i}$ is in the rows corresponding to those where the blocks $A_{i-1}$ and $G_{i-1}$ are positioned in $T_{d}^{\prime}$ ), by Lemma 2.3 again; since the columns of $G_{i-1}$ include the columns of $A_{i-1} G_{i}$, it follows that $\mathbf{s} \stackrel{T_{d}^{\prime}}{\longrightarrow}+0$ in this case also.

Finally, suppose that $\mathbf{f}$ and $\mathbf{g}$ have leading terms in the last row of $T_{d}^{\prime}$. In this case, either $\mathbf{s} \stackrel{T_{d}^{\prime}}{\longrightarrow}+0$ because the columns of $G_{1}$ form a Gröbner basis, or $\mathbf{s}=S\left(X \mathbf{e}_{\left(\begin{array}{c}d-1 \\ 2\end{array}\right)}, h \mathbf{e}_{\left(\begin{array}{c}d-1 \\ 2\end{array}\right)}\right)$ for some $h \in L[Y, Z]$; in the latter case, s reduces to 0 in one step modulo $\left\{X \mathbf{e}_{\left(\begin{array}{c}d-1 \\ 2\end{array}\right)}\right\}$.

Thus, in all cases, $\mathbf{s} \stackrel{T_{d}^{\prime}}{\longrightarrow}+0$. Hence, by (the analogue of) [A-L, Theorem 3.5.19], the columns of $T_{d}^{\prime}$ form a Gröbner basis.

To complete the proof of part (i), it only remains for us to show that $\operatorname{Im} T_{d}^{\prime}=$ $\operatorname{Im} T_{d}$. It is easy to see by descending induction on $i$ that, for all $i=d-2, d-3$, $\ldots, 1$, the columns of $G_{i}$ include the columns of $A_{i} A_{i+1} \ldots A_{d-2}$ and form a Gröbner basis (over $L[Y, Z]$ ) for $\operatorname{Im} A_{i} A_{i+1} \ldots A_{d-2}$; hence (over both $L[Y, Z]$ and $L[X, Y, Z])$

$$
\operatorname{Im} A_{i} A_{i+1} \ldots A_{d-2}=\operatorname{Im} A_{i} G_{i+1}=\operatorname{Im} G_{i} \quad \text { for all } i=d-3, d-4, \ldots, 1 .
$$

By Lemma 2.3 for such an $i$, each column of

$$
\left[\begin{array}{c}
0 \\
\vdots \\
0 \\
A_{i} G_{i+1} \\
0 \\
\vdots \\
0
\end{array}\right]
$$

(in which the block $A_{i} G_{i+1}$ occupies the rows corresponding to those occupied by $A_{i}$ in $T_{d}$ ) can by obtained as a result of reducing modulo $T_{d}$ the $S$-polynomial of a 
column of $T_{d}$ and a column of

$$
\left[\begin{array}{c}
0 \\
\vdots \\
0 \\
G_{i+1} \\
0 \\
\vdots \\
0
\end{array}\right]
$$

(in which the block $G_{i+1}$ occupies the rows corresponding to those occupied by $A_{i+1}$ in $T_{d}$ ). The claim in part (i) now follows from another use of descending induction.

(ii) Since the lexicographical order we are using on $L[X, Y, Z]$ is an elimination order with $X$ greater than $Y$ and $Z$, it follows from (the analogue of) A-L, Theorem 3.6.6] that the intersection of the set of columns of $T_{d}^{\prime}$ with $L[Y, Z]^{\left(\begin{array}{c}d-1 \\ 2\end{array}\right)}$ provides a

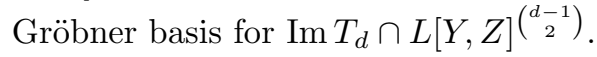

Therefore the columns of

$$
\left[\begin{array}{c|ccccc}
A_{d-2} & G_{d-2} & 0 & \ldots & & 0 \\
0 & 0 & G_{d-3} & 0 & \cdots & 0 \\
0 & 0 & 0 & G_{d-4} & & 0 \\
\vdots & \vdots & \vdots & & \ddots & \vdots \\
0 & 0 & 0 & \cdots & 0 & G_{1}
\end{array}\right]
$$

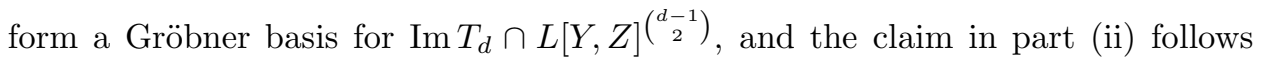
from this.

The following lemma provides motivation for part (ii) of the above theorem.

2.5. Lemma. Consider the matrices $T_{d}$ of 2.2 and $H_{d}$ of 2.4 . Let $r \in L \backslash\{0\}$. Then $r$ annihilates a non-zero element of Coker $T_{d}$ if and only if $r$ annihilates a

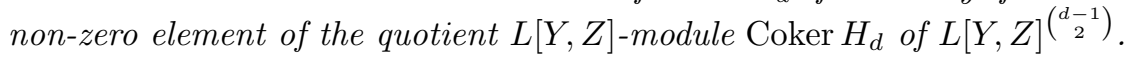

Proof. Suppose that $r$ annihilates a non-zero element of Coker $T_{d}$. Thus there exists a $v \in R_{0}^{\left(\frac{d-1}{2}\right)} \backslash \operatorname{Im} T_{d}$ such that $r v \in \operatorname{Im} T_{d}$. We can and do assume that $v$ has been chosen so that its leading term is minimal among the leading terms of all possible such columns. But $X \mathbf{e}_{1}, X \mathbf{e}_{2}, \ldots, X \mathbf{e}_{\left(\begin{array}{c}d-1 \\ 2\end{array}\right)}$ are all leading terms of columns of $T_{d}$, and so $v$ does not involve $X$. In view of this and the fact, established in 2.4, that $\operatorname{Im} H_{d} \subseteq \operatorname{Im} T_{d}$, we have $v \in L[Y, Z]^{\left(\begin{array}{c}-1 \\ 2\end{array}\right)} \backslash \operatorname{Im} H_{d}$. Furthermore, $r v \in$ $\operatorname{Im} T_{d} \cap L[Y, Z]^{\left(\frac{d-1}{2}\right)}$, and, by 2.4 this is the $L[Y, Z]$-submodule of $L[Y, Z]^{\left(\begin{array}{c}d-1 \\ 2\end{array}\right)}$ generated by the columns of $H_{d}$.

The converse is even easier.

2.6. Proposition. For each integer $i=0, \ldots, n-1, A_{i+1} A_{i+2} \ldots A_{n}$ is the $(i+1) \times(n+1)$ matrix

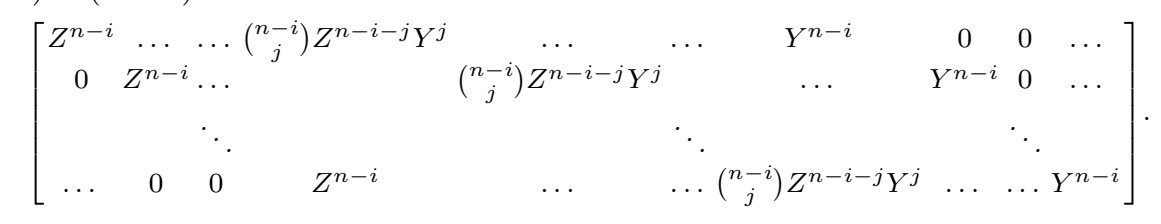


Proof. The result follows from an easy reverse induction on $i$.

The particular case of 2.6 in which $i=0$ yields the following.

2.7. Corollary. $A_{1} A_{2} \ldots A_{n}$ is the $1 \times(n+1)$ matrix whose $(1, i+1)$-th entry is $\left(\begin{array}{c}n \\ i\end{array}\right) Y^{i} Z^{n-i}$ for all $i=0, \ldots, n$.

2.8. Proposition. Let $r, k \in \mathbb{N}$, and let $Q_{r, r+k}$ be the $r \times(r+k)$ matrix with entries in $L[Y, Z]$ given by

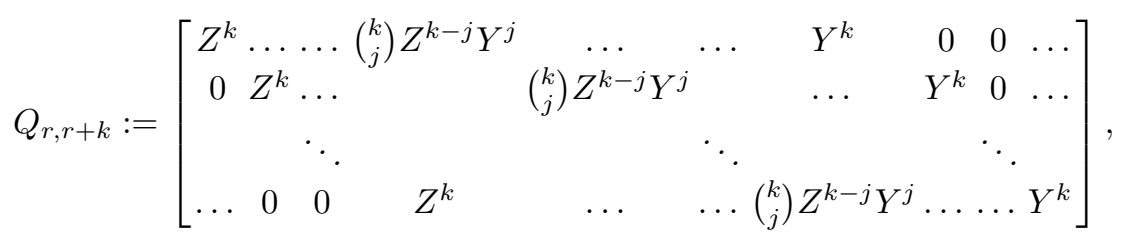

let $\mathbf{c}_{j}$ denote the $j$-th column of $Q_{r, r+k}($ for $j=1, \ldots, r+k)$, and let $\widetilde{Q}_{r, r+k}$ be the result of evaluation of $Q_{r, r+k}$ at $Y=Z=1$. Thus

$$
\widetilde{Q}_{r, r+k}:=\left[\begin{array}{cccccccccc}
1 & \ldots & \ldots & \left(\begin{array}{c}
k \\
j
\end{array}\right) & \ldots & \ldots & 1 & 0 & 0 & \ldots \\
0 & 1 & \ldots & & \left(\begin{array}{l}
k \\
j
\end{array}\right) & & \ldots & 1 & 0 & \ldots \\
& & \ddots & & & \ddots & & & \ddots & \\
\ldots & 0 & 0 & 1 & \ldots & \ldots & \left(\begin{array}{c}
k \\
j
\end{array}\right) & \ldots & \ldots & 1
\end{array}\right] .
$$

Consider $L[Y, Z]$ as an $\mathbb{N}_{0}{ }^{2}$-graded ring in which $L[Y, Z]_{(0,0)}=L$ and $\operatorname{deg} Y^{i} Z^{j}=$ $(i+j, i)$. Turn the free $L[Y, Z]$-module

$$
L[Y, Z]^{r}=L[Y, Z] \mathbf{e}_{1} \oplus \cdots \oplus L[Y, Z] \mathbf{e}_{r}
$$

into an $\mathbb{N}_{0}{ }^{2}$-graded module over the $\mathbb{N}_{0}{ }^{2}$-graded ring $L[Y, Z]$ in such a way that $\operatorname{deg} \mathbf{e}_{i}=(0, i)$ for $i=1, \ldots, r$. All references to gradings in the rest of this proposition and its proof refer to this $\mathbb{N}_{0}{ }^{2}$-grading.

(i) For all $i \in \mathbb{N}_{0}$ and $j \in \mathbb{N}$, the component $\left(L[Y, Z]^{r}\right)_{(i, j)}$ is a free L-module with base

$$
\left(Y^{j-\rho} Z^{i-j+\rho} \mathbf{e}_{\rho}\right)_{\rho=\max \{j-i, 1\}, \ldots, \min \{j, r\}} \cdot
$$

(Of course, we interpret a free module with an empty base as 0. )

(ii) $\operatorname{Im} Q_{r, r+k}$ is a graded submodule of $L[Y, Z]^{r}$, and, for all $i \in \mathbb{N}_{0}, j \in \mathbb{N}$,

$$
\left(\operatorname{Im} Q_{r, r+k}\right)_{(i, j)}= \begin{cases}0 & \text { if } i<k, \\ \sum_{\sigma=\max \{j+k-i, 1\}}^{\min \{j, r+k\}} L Y^{j-\sigma} Z^{i-j+\sigma-k} \mathbf{c}_{\sigma} & \text { if } i \geq k, \\ \left(L[Y, Z]^{r}\right)_{(i, j)} & \text { if } i \geq 2 k+r \\ & \text { or } j \geq k+r .\end{cases}
$$

(iii) The $\mathbb{N}_{0}{ }^{2}$-graded $L[Y, Z]$-module Coker $Q_{r, r+k}$ vanishes in all except finitely many degrees; in fact, Coker $Q_{r, r+k}$ is a finitely generated L-module with

$$
\text { Coker } Q_{r, r+k}=\bigoplus_{i=0}^{2 k+r-1} \bigoplus_{j=1}^{k+r-1}\left(\operatorname{Coker} Q_{r, r+k}\right)_{(i, j)} ;
$$

for $0 \leq i<k$ (and $j \in \mathbb{N}$ ), the component $\left(\text { Coker } Q_{r, r+k}\right)_{(i, j)}$ is a free $L$ module; and for $k \leq i \leq 2 k+r-1$ and $1 \leq j \leq k+r-1$, the component 
$\left(\text { Coker } Q_{r, r+k}\right)_{(i, j)}$, as an L-module, is isomorphic to the cokernel of a submatrix of $\widetilde{Q}_{r, r+k}$ made up of the (consecutive) columns of that matrix numbered $\max \{j+k-i, 1\}, \max \{j+k-i, 1\}+1, \ldots, \min \{j, r+k\}$.

Proof. (i) This is immediate from the fact that, for $\alpha, \beta \in \mathbb{N}_{0}$ and $\rho \in\{1, \ldots, r\}$, we have $\operatorname{deg} Y^{\alpha} Z^{\beta} \mathbf{e}_{\rho}=(\alpha+\beta, \alpha+\rho)$.

(ii) Note that $\mathbf{c}_{j}$ is a homogeneous element of $L[Y, Z]^{r}$ of degree $(k, j)$ (for all $j=1, \ldots, k+r)$. Hence $\operatorname{Im} Q_{r, r+k}$ is a graded submodule of $L[Y, Z]^{r}$, and a homogeneous element of $\operatorname{Im} Q_{r, r+k}$ is expressible as an $L[Y, Z]$-linear combination of the columns of $Q_{r, r+k}$ in which all the coefficients are homogeneous. Note that $\operatorname{deg} Y^{\alpha} Z^{\beta} \mathbf{c}_{\sigma}=(\alpha+\beta+k, \alpha+\sigma)$ (for $\alpha, \beta \in \mathbb{N}_{0}$ and $\left.\sigma \in\{1, \ldots, r\}\right)$. Hence

$$
\left(\operatorname{Im} Q_{r, r+k}\right)_{(i, j)}=0
$$

if $i<k$, while

$$
\left(\operatorname{Im} Q_{r, r+k}\right)_{(i, j)}=\sum_{\sigma=\max \{j+k-i, 1\}}^{\min \{j, r+k\}} L Y^{j-\sigma} Z^{i-j+\sigma-k} \mathbf{c}_{\sigma}
$$

if $i \geq k$.

Notice that the vectors $Z^{k} \mathbf{e}_{1}, Z^{k+1} \mathbf{e}_{2}, \ldots, Z^{k+r-1} \mathbf{e}_{r}$ and $Y^{k+r-1} \mathbf{e}_{1}, Y^{k+r-2} \mathbf{e}_{2}$, $\ldots, Y^{k} \mathbf{e}_{r}$ are all in $\operatorname{Im} Q_{r, r+k}$ : for any $1<s \leq r$ multiply the $s$-th column of $Q_{r, r+k}$ by $Z^{s-1}$ and reduce with respect to $Z^{k} \mathbf{e}_{1}, Z^{k+1} \mathbf{e}_{2}, \ldots, Z^{k+s-2} \mathbf{e}_{s-1}$ to obtain $Z^{k+s-1} \mathbf{e}_{s} \in \operatorname{Im} Q_{r, r+k}$; a similar argument shows that

$$
Y^{k} \mathbf{e}_{r}, Y^{k+1} \mathbf{e}_{r-1}, \ldots, Y^{k+r-1} \mathbf{e}_{1} \in \operatorname{Im} Q_{r, r+k} .
$$

Therefore, if $i \geq 2 k+r$ or $j \geq k+r$, then

$$
Y^{j-\rho} Z^{i-j+\rho} \mathbf{e}_{\rho} \in \operatorname{Im} Q_{r, r+k} \quad \text { for all } \rho=\max \{j-i, 1\}, \ldots, \min \{j, r\}
$$

since $Y^{j-\rho} \mathbf{e}_{\rho} \in \operatorname{Im} Q_{r, r+k}$ if $j \geq k+r$, while if $j<k+r$ and $i \geq 2 k+r$, then $i-j \geq k+1$ and $Z^{i-j+\rho} \mathbf{e}_{\rho} \in \operatorname{Im} Q_{r, r+k}$. Thus, for $i \geq 2 k+r$ or $j \geq k+r$, all the members of the base found in part (i) for the free $L$-module $L[Y, Z]_{(i, j)}^{r}$ lie in $\operatorname{Im} Q_{r, r+k}$.

(iii) All the claims of this except the final one now follow from the previous parts. To deal with the final one, suppose that $k \leq i \leq 2 k+r-1$ and $1 \leq j \leq k+r-1$. We shall use 'overlines' to denote natural images in cokernels of elements of free modules. It will be convenient to abbreviate $\min \{j, r+k\}$ by $\gamma$ and $\max \{j+k-i, 1\}$ by $\beta$; the conditions imposed on $i$ and $j$ ensure that $\beta \leq \gamma$.

By part (i), $\left(\text { Coker } Q_{r, r+k}\right)_{(i, j)}$ is generated, as an $L$-module, by

$$
\left\{\overline{Y^{j-\rho} Z^{i-j+\rho} \mathbf{e}_{\rho}}: \rho=\max \{j-i, 1\}, \ldots, \min \{j, r\}\right\} .
$$

(Note that, once again, the conditions imposed on $i$ and $j$ ensure that

$$
\max \{j-i, 1\} \leq \min \{j, r\} \text {.) }
$$

The fact that, for each $\sigma=\beta, \ldots, \gamma$, we have

$$
\overline{Y^{j-\sigma} Z^{i-j+\sigma-k} \mathbf{c}_{\sigma}}=0
$$

shows that the $\sigma$-th column of $\widetilde{Q}_{r, r+k}$ leads to a column of relations on the generators displayed above. Furthermore, part (ii) shows that every column of relations on those generators is an $L$-linear combination of the columns of relations arising (in this way) from the $\beta$-th, $(\beta+1)$-th, $\ldots, \gamma$-th columns of $\widetilde{Q}_{r, r+k}$. 
2.9. Remark. Note that, with the notation of 2.8 (and provided $r>1$ ), we have

$$
Q_{r-1, r+k}=Q_{r-1, r} Q_{r, r+k} .
$$

2.10. Remark. Let $B$ be a matrix with integer entries and positive rank $d$; let $p$ be a prime number. Then it follows from the theory of the Smith normal form that $p \mathbb{Z} \in \operatorname{Ass}_{\mathbb{Z}}($ Coker $B)$ if and only if the ideal generated by the $d \times d$ minors of $B$ is contained in $p \mathbb{Z}$.

In view of Remark 2.10] and Proposition 2.8 we are going, in the case when $L=\mathbb{Z}$, to be interested in the value of the determinant of a square matrix (with integer entries) of the form

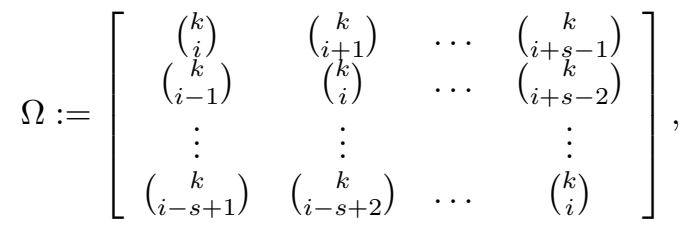

where $k, s \in \mathbb{N}, i \in \mathbb{N}_{0}$ and we use the convention that a binomial coefficient $\left(\begin{array}{l}\xi \\ \eta\end{array}\right)$ is 0 if either $\eta<0$ or $\eta>\xi$. The value of this determinant was known to V. van Zeipel in 1865 [Z]; the calculation is described in [Mu, Chapter XX]. For the convenience of the reader, we indicate a route to the answer.

2.11. Proposition (see van Zeipel [Z]). Let $\Omega$ be as displayed above. Then

$$
\operatorname{det} \Omega=\prod_{j=0}^{s-1} \frac{\left(\begin{array}{c}
k+s-1-j \\
i
\end{array}\right)}{\left(\begin{array}{c}
i+j \\
i
\end{array}\right)} \text {. }
$$

Proof. Add the penultimate row of $\Omega$ to the last row; in the result, add the $(r-2)$ th row to the $(r-1)$-th, and continue in this way until the first row has been added to the second. In this way one sees that

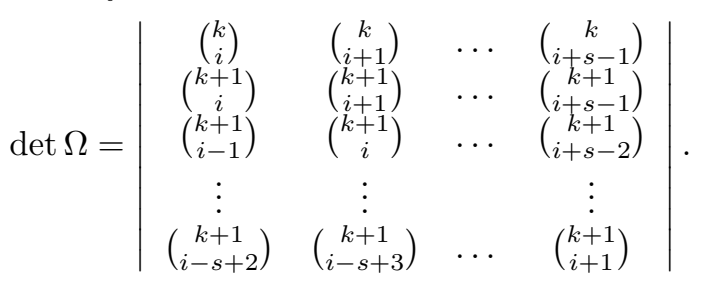

Now repeat the same sequence of elementary row operations, but this time stop after the second row has been added to the third; then do a further such sequence, this time stopping after the third row has been added to the fourth. Continue in this way to see that

$$
\operatorname{det} \Omega=\left|\begin{array}{cccc}
\left(\begin{array}{c}
k \\
i
\end{array}\right) & \left(\begin{array}{c}
k \\
i+1
\end{array}\right) & \ldots & \left(\begin{array}{c}
k \\
i+s-1 \\
k+1
\end{array}\right) \\
(k+1 \\
i \\
k & \left(\begin{array}{c}
k+1 \\
i+1
\end{array}\right) & \ldots & \left(\begin{array}{c}
k+1 \\
i+s-1
\end{array}\right) \\
\left(\begin{array}{c}
k+2 \\
i
\end{array}\right) & \left(\begin{array}{c}
k+2 \\
i+1
\end{array}\right) & \ldots & \left(\begin{array}{c}
k+2 \\
i+s-1
\end{array}\right) \\
\vdots & \vdots & & \vdots \\
\left(\begin{array}{c}
k+s-1 \\
i
\end{array}\right) & \left(\begin{array}{c}
k+s-1 \\
i+1
\end{array}\right) & \ldots & \left(\begin{array}{c}
k+s-1 \\
i+s-1
\end{array}\right)
\end{array}\right| .
$$

We proceed by induction on $i$. When $i=0$, it is clear from the initial form of $\Omega$ that $\operatorname{det} \Omega=1$, and the claim is true. We therefore assume that $i>0$, and make the obvious inductive assumption. 
With reference to the last display, take out a factor $k+j-1$ from the $j$-th row $(1 \leq j \leq s)$ and a factor $1 /(i+l-1)$ from the $l$-th column $(1 \leq l \leq s)$ to see that

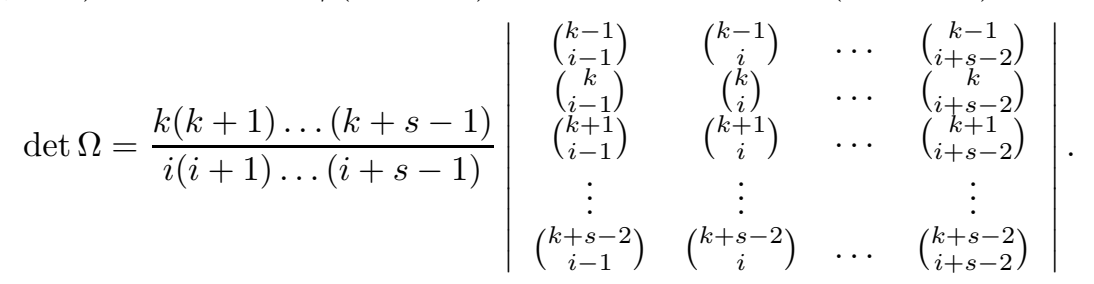

Now use the inductive hypothesis.

2.12. Corollary. In the situation of Proposition 2.11, we have

$$
\operatorname{det} \Omega=\frac{\prod_{j=0}^{s-1}\left(\begin{array}{c}
k+s-1 \\
i+j
\end{array}\right)}{\prod_{j=0}^{s-1}\left(\begin{array}{c}
k+s-1 \\
j
\end{array}\right)} \text {. }
$$

Proof. First note that, for $j \in\{1, \ldots, s-1\}$, we have

$$
\left(\begin{array}{c}
k+s-1-j \\
i
\end{array}\right)=\left(\begin{array}{c}
k+s-1 \\
i+j
\end{array}\right) \frac{(i+1) \ldots(i+j)}{(k+s-j) \ldots(k+s-1)} .
$$

It therefore follows from Proposition 2.11 that

$$
\begin{aligned}
\operatorname{det} \Omega & =\prod_{j=0}^{s-1} \frac{\left(\begin{array}{c}
k+s-1-j \\
i
\end{array}\right)}{\left(\begin{array}{c}
i+j \\
i
\end{array}\right)} \\
& =\left(\prod_{j=0}^{s-1}\left(\begin{array}{c}
k+s-1 \\
i+j
\end{array}\right) \frac{i ! j !}{i !}\right)\left(\prod_{j=0}^{s-1} \frac{1}{(k+s-j) \ldots(k+s-1)}\right) \\
& =\left(\begin{array}{c}
s-1 \\
j=0
\end{array}\left(\begin{array}{c}
k+s-1 \\
i+j
\end{array}\right)\right)\left(\prod_{j=0}^{s-1} \frac{j !(k+s-j-1) !}{(k+s-1) !}\right) \\
& =\frac{\prod_{j=0}^{s-1}\left(\begin{array}{c}
k+s-1 \\
i+j
\end{array}\right)}{\prod_{j=0}^{s-1}\left(\begin{array}{c}
k+s-1 \\
j
\end{array}\right)}
\end{aligned}
$$

2.13. Notation. For each $n \in \mathbb{N}$, we set

$$
\Pi(n):=\left\{p: p \text { is a prime factor of }\left(\begin{array}{c}
n \\
i
\end{array}\right) \text { for some } i \in\{0, \ldots, n\}\right\} .
$$

2.14. Corollary. Let $r, k \in \mathbb{N}$ and consider the matrix $\widetilde{Q}_{r, r+k}$ of 2.8 , Let $\Delta$ be a submatrix of $\widetilde{Q}_{r, r+k}$ formed by $c(>0)$ consecutive columns of that matrix; set $s:=\min \{c, r\}$. If $p \in \mathbb{Z}$ is a prime number such that every $s \times s$ minor of $\Delta$ is contained in $p \mathbb{Z}$, then $p \in \Pi(r+k-1)$.

Proof. We argue by induction on $r$. Note that $\widetilde{Q}_{1,1+k}$ is the $1 \times(1+k)$ matrix

$$
\left[\begin{array}{llllll}
1 & \left(\begin{array}{l}
k \\
1
\end{array}\right) & \ldots & \left(\begin{array}{l}
k \\
j
\end{array}\right) & \ldots & 1
\end{array}\right],
$$

and so the result is clear in this case.

Now suppose that $r>1$ and that the result has been established, for all values of $k$, for smaller values of $r$. 
If $s=r$, then there is an $r \times r$ submatrix of $\widetilde{Q}_{r, r+k}$ of the form

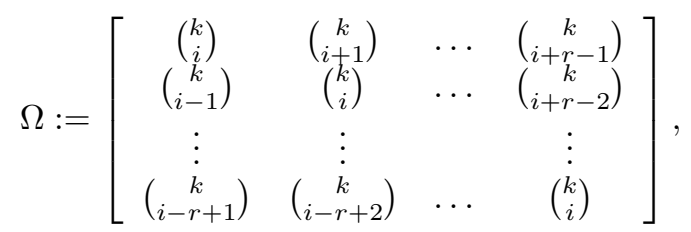

where $i \in\{0, \ldots, k\}$, such that $\operatorname{det} \Omega \in p \mathbb{Z}$. It now follows from Corollary[2.12 that $p$ is a factor of $\left(\begin{array}{c}k+r-1 \\ l\end{array}\right)$ for some $l \in\{0, \ldots, k+r-1\}$.

Now suppose that $s=c<r$. Set $D^{\prime}:=\widetilde{Q}_{r-1, r} D$. As $\widetilde{Q}_{r-1, r+k}=\widetilde{Q}_{r-1, r} \widetilde{Q}_{r, r+k}$ by 2.9, it follows that $\Delta^{\prime}:=\widetilde{Q}_{r-1, r} \Delta$ is the $(r-1) \times c$ submatrix of $\widetilde{Q}_{r-1, r+k}$ involving the same columns as $\Delta$. But

$$
\widetilde{Q}_{r-1, r}=\left[\begin{array}{ccccc}
1 & 1 & 0 & \ldots & 0 \\
0 & 1 & 1 & 0 & \ldots \\
\vdots & & \ddots & \ddots & \\
0 & \ldots & 0 & 1 & 1
\end{array}\right]
$$

and so the rows of $\Delta^{\prime}$ are the sums of consecutive rows of $\Delta$. Therefore any $s \times s$ minor of $\Delta^{\prime}$ is the sum of $2^{s}$ determinants, each one being either obviously zero or an $s \times s$ minor of $\Delta$. Hence every $s \times s$ minor of $\Delta^{\prime}$ is contained in $p \mathbb{Z}$, and so, by the inductive hypothesis, $p \in \Pi(r-1+k+1-1)$, that is, $p \in \Pi(r+k-1)$.

2.15. Lemma. The set of integers $\{\# \Pi(n): n \in \mathbb{N}\}$ is unbounded.

Proof. Let $\left(p_{n}\right)_{n \in \mathbb{N}}$ be an enumeration of the prime numbers. Then, for each $n \in \mathbb{N}$, we have

$$
p_{1} p_{2} \ldots p_{n}=\left(\begin{array}{c}
p_{1} p_{2} \ldots p_{n} \\
1
\end{array}\right) \in \Pi\left(p_{1} p_{2} \ldots p_{n}\right)
$$

2.16. Lemma. Let $p \in \mathbb{Z}$ be a prime number. Then the sets

$$
\{j \in \mathbb{N}: j \geq 3 \text { and } p \in \Pi(j-2)\} \quad \text { and } \quad\{j \in \mathbb{N}: j \geq 3 \text { and } p \notin \Pi(j-2)\}
$$

are both infinite.

Proof. If $p$ divides $j-2 \in \mathbb{N}$, then $p \in \Pi(j-2)$ because $p$ divides $\left(\begin{array}{c}j-2 \\ 1\end{array}\right)=j-2$; hence the first set is infinite.

To prove that the second set is infinite it is enough to show that $p \notin \Pi\left(p^{k}-1\right)$ for all $k \geq 1$. Let $T$ be an indeterminate; working modulo $p$ we have

$$
(1+T)^{p^{k}-1}(1+T)=(1+T)^{p^{k}} \equiv 1+T^{p^{k}},
$$

and if we compare the coefficients of $T^{i}$ on both sides of this congruence we see that, for $0<i \leq p^{k}-1$,

$$
\left(\begin{array}{c}
p^{k}-1 \\
i
\end{array}\right)+\left(\begin{array}{c}
p^{k}-1 \\
i-1
\end{array}\right) \equiv 0
$$

and since $p$ does not divide $\left(\begin{array}{c}p^{k}-1 \\ 0\end{array}\right)=1$, an easy induction on $i$ shows that $p$ does not divide $\left(\begin{array}{c}p^{k}-1 \\ i\end{array}\right)$ for all $i$ with $0 \leq i \leq p^{k}-1$.

We are now ready to present our main results about Singh's example. 
2.17. Theorem. Let $R^{\prime}$ denote the ring $\mathbb{Z}[X, Y, Z, U, V, W] /(X U+Y V+Z W)$ (considered by Singh) graded in the manner described in 2.1; let $-d \in \mathbb{Z}$ with $d \geq 3$; and let $p \in \mathbb{Z}$ be a prime number. Then:

(i) $p \mathbb{Z} \in \operatorname{Ass}_{\mathbb{Z}}\left(H_{R_{+}^{\prime}}^{3}\left(R^{\prime}\right)_{-d}\right)$ if and only if $p \in \Pi(d-2)$;

(ii) $\operatorname{Ass}_{R_{0}^{\prime}}\left(H_{R_{+}^{\prime}}^{3}\left(R^{\prime}\right)_{-d}\right)=\{(X, Y, Z)\} \cup\{(q, X, Y, Z): q \in \Pi(d-2)\}$;

(iii) the set of integers $\left\{\#\left(\operatorname{Ass}_{R_{0}^{\prime}}\left(H_{R_{+}^{\prime}}^{3}\left(R^{\prime}\right)_{-j}\right)\right): j \geq 3\right\}$ is unbounded;

(iv) the sets

$$
\left\{j \in \mathbb{Z}: j \geq 3 \text { and }(p, X, Y, Z) \in \operatorname{Ass}_{R_{0}}^{\prime}\left(H_{R_{+}^{\prime}}^{3}\left(R^{\prime}\right)_{-j}\right)\right\}
$$

and

$$
\left\{j \in \mathbb{Z}: j \geq 3 \text { and }(p, X, Y, Z) \notin \operatorname{Ass}_{R_{0}}^{\prime}\left(H_{R_{+}^{\prime}}^{3}\left(R^{\prime}\right)_{-j}\right)\right\}
$$

are both infinite; and

(v) $\operatorname{Ass}_{R_{0}^{\prime}}\left(H_{R_{+}^{\prime}}^{3}\left(R^{\prime}\right)_{n}\right)$ is not asymptotically increasing for $n \rightarrow-\infty$.

Proof. (i) It follows from Lemma 2.2 that $p \mathbb{Z} \in \operatorname{Ass}_{\mathbb{Z}}\left(H_{R_{+}^{\prime}}^{3}\left(R^{\prime}\right)_{-d}\right)$ if and only if $p \mathbb{Z} \in \operatorname{Ass}_{\mathbb{Z}}\left(\right.$ Coker $\left.T_{d}\right)$; furthermore, by Lemma 2.5, this is the case if and only if $p \mathbb{Z} \in$ $\operatorname{Ass}_{\mathbb{Z}}\left(\right.$ Coker $H_{d}$ ), where the matrix $H_{d}$ is as defined in Theorem 2.4. It therefore follows from 2.6 and the notation introduced in 2.8 that $p \mathbb{Z} \in \operatorname{Ass}_{\mathbb{Z}}\left(H_{R_{+}^{\prime}}^{3}\left(R^{\prime}\right)_{-d}\right)$ if and only if

$$
p \mathbb{Z} \in \bigcup_{i=1}^{d-2} \operatorname{Ass}_{\mathbb{Z}}\left(\operatorname{Coker} Q_{i, d-1}\right) .
$$

Suppose that $p \in \Pi(d-2)$, so that there exists $j \in\{1, \ldots, d-3\}$ such that $p$ is a factor of $\left(\begin{array}{c}d-2 \\ j\end{array}\right)$. Then it follows from Theorem 2.8 (iii) that (for example) $p \mathbb{Z} \in \operatorname{Ass}_{\mathbb{Z}}\left(\operatorname{Coker} Q_{1, d-1}\right)_{d-2, j+1}$.

Conversely, suppose that $p \mathbb{Z} \in \operatorname{Ass}_{\mathbb{Z}}\left(\operatorname{Coker} Q_{i, d-1}\right)$, where $i \in\{1, \ldots, d-2\}$. We use Theorem 2.8(iii) to see that $p \mathbb{Z} \in \operatorname{Ass}_{\mathbb{Z}}(\operatorname{Coker} \Delta)$, where $\Delta$ is a submatrix of $\widetilde{Q}_{i, d-1}$ formed by $c(>0)$ consecutive columns of that matrix; set $s:=\min \{c, i\}$. It follows from Proposition 2.11 that $\Delta$ has rank $s$, and therefore from Remark 2.10 that the ideal generated by the $s \times s$ minors of $\Delta$ is contained in $p \mathbb{Z}$. Therefore $p \in \Pi(d-2)$ by Corollary 2.14 .

(ii) This is now immediate from part (i) and Lemma 2.2 ,

(iii) This is a consequence of part (ii) and Lemma 2.15

(iv) This is now immediate from part (ii) and Lemmas 2.2 and 2.16

(v) This is a consequence of parts (ii) and (iv).

\section{REFERENCES}

[A-L] W. W. Adams and P. Loustaunau, An introduction to Gröbner bases, American Mathematical Society, Providence, Rhode Island, 1994. MR 95g:13025

[B] M. Brodmann, A lifting result for local cohomology of graded modules, Math. Proc. Cambridge Philos. Soc. 92 (1982), 221-229. MR 84c:13014.

[B-H] M. Brodmann and M. Hellus, Cohomological patterns of coherent sheaves over projective schemes, J. Pure and Appl. Algebra, to appear.

[B-M-M] M. Brodmann, C. Matteotti and Nguyen Duc Minh, Bounds for cohomological Hilbert functions of projective schemes over Artinian rings, Vietnam J. Math. 28 (2000), 341380. MR 2001j:14022 
[B-S] M. P. Brodmann and R. Y. Sharp, Local cohomology: an algebraic introduction with geometric applications, Cambridge University Press, 1998. MR 99h:13020

[G] A. Grothendieck, Cohomologie locale des faisceaux cohérents et théorèmes de Lefschetz locaux et globaux (SGA 2), Séminaire de Géométrie Algébrique du Bois-Marie 1962, North-Holland, Amsterdam, 1968. MR 57:16294

[Ma] H. Matsumura, Commutative Algebra, Benjamin, New York, 1970. MR 42:1813

$[\mathrm{Mu}] \mathrm{T}$. Muir, The theory of determinants in the historical order of development, Volume III, Macmillan, London, 1920

[S] R. Y. Sharp, Bass numbers in the graded case, a-invariant formulas, and an analogue of Faltings' Annihilator Theorem, J. Algebra 222 (1999), 246-270. MR 2000j:13027

[Si] A. K. Singh, $p$-torsion elements in local cohomology modules, Math. Research Letters 7 (2000) 165-176. MR 2001g:13039

[Z] V. van Zeipel, Om determinanter, hvars elementer äro binomialkoefficienter, Lunds Universitet Årsskrift ii (1865) 1-68.

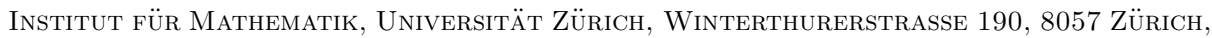
SWITZERLAND

E-mail address: Brodmann@math.unizh.ch

Department of Pure Mathematics, University of Sheffield, Hicks Building, Sheffield S3 7RH, United KINGDOM

E-mail address: M.Katzman@sheffield.ac.uk

Department of Pure Mathematics, University of Sheffield, Hicks Building, Sheffield S3 7RH, UNITED KINGDOM

E-mail address: R.Y.Sharp@sheffield.ac.uk 